\title{
Comparison of particle trajectories and collision operators for collisional transport in nonaxisymmetric plasmas
}

\author{
M. Landreman, ${ }^{1, a)}$ H. M. Smith, ${ }^{2}$ A. Mollén, ${ }^{3}$ and P. Helander ${ }^{2}$ \\ ${ }^{1}$ Institute for Research in Electronics and Applied Physics, University of Maryland, College Park, \\ Maryland 20742, USA \\ ${ }^{2}$ Max-Planck-Institut für Plasmaphysik, 17491 Greifswald, Germany \\ ${ }^{3}$ Department of Applied Physics, Chalmers University of Technology, Göteborg, Sweden
}

(Received 20 December 2013; accepted 18 March 2014; published online 4 April 2014)

\begin{abstract}
In this work, we examine the validity of several common simplifying assumptions used in numerical neoclassical calculations for nonaxisymmetric plasmas, both by using a new continuum drift-kinetic code and by considering analytic properties of the kinetic equation. First, neoclassical phenomena are computed for the LHD and W7-X stellarators using several versions of the drift-kinetic equation, including the commonly used incompressible- $E \times B$-drift approximation and two other variants, corresponding to different effective particle trajectories. It is found that for electric fields below roughly one third of the resonant value, the different formulations give nearly identical results, demonstrating the incompressible $E \times B$-drift approximation is quite accurate in this regime. However, near the electric field resonance, the models yield substantially different results. We also compare results for various collision operators, including the full linearized Fokker-Planck operator. At low collisionality, the radial transport driven by radial gradients is nearly identical for the different operators; while in other cases, it is found to be important that collisions conserve momentum. (C 2014 AIP Publishing LLC. [http://dx.doi.org/10.1063/1.4870077]
\end{abstract}

\section{INTRODUCTION}

One important difference between axisymmetric and nonaxisymmetric plasmas is that neoclassical effects in the latter are more sensitive to small values of the radial electric field $E_{r}$. In axisymmetric plasmas, in order for the radial electric to modify the collisional ion heat flux and other neoclassical phenomena, the poloidal ion Mach number $\left(B / B_{\text {pol }}\right)\left|\boldsymbol{v}_{E}\right| / v_{\text {i }}$ must approach $\sim 1$, since an $E_{r}$ of corresponding magnitude is required to modify the trapped region of phase space. ${ }^{1}$ Here, $B$ is the magnetic field magnitude, $B_{\text {pol }}$ is the poloidal magnetic field, $\boldsymbol{v}_{E}$ is the $\boldsymbol{E} \times \boldsymbol{B}$ drift, and $v_{\mathrm{i}}=\sqrt{2 T_{\mathrm{i}} / m_{\mathrm{i}}}$ is the ion thermal speed. However, in nonaxisymmetric plasmas, a much smaller value of $E_{r}$ can modify the collisional fluxes. ${ }^{2-4}$ The reason is that helically trapped particles experience a secular radial magnetic drift, and whichever process first interrupts this radial motion will thereby determine the step size for radial diffusion. When $E_{r}=0$, the radial magnetic drift is interrupted by collisions, which cause the particle to gain parallel momentum and de-trap. But if $E_{r}$ is sufficient for the poloidal $\boldsymbol{E} \times \boldsymbol{B}$ precession frequency to exceed the effective collisional detrapping rate, $\boldsymbol{E} \times \boldsymbol{B}$ precession begins to carry helically trapped particles onto untrapped trajectories, and also confines the trapped orbits by convecting them (usually poloidally) around the torus, thereby limiting the radial step size and transport. This transition from collisional $(1 / \nu$-regime) to $E_{r}$-limited $(\sqrt{\nu}$-regime $)$ transport typically occurs at values of $E_{r}$ for which the poloidal Mach number is still $\ll 1$, due to the low collisionality in typical experiments. (Here, $\nu$ denotes a collision frequency.) For this reason, stellarator

\footnotetext{
a)mattland@umd.edu
}

transport at low collisionality is sensitive to small values of $E_{r}$. A variety of codes have been developed to compute these neoclassical effects in stellarators. ${ }^{5-13}$

However, including the physics of $\boldsymbol{E} \times \boldsymbol{B}$ precession in a $\delta f$ drift-kinetic equation (or code to solve such an equation) is complicated by several issues. First, if a rigorous expansion in $\rho_{*} \ll 1$ is employed, $\boldsymbol{E} \times \boldsymbol{B}$ precession is formally excluded when the usual drift ordering $\boldsymbol{v}_{E} \sim \rho_{*} v_{\text {i }}$ is used, but the high-flow ordering $\boldsymbol{v}_{E} \sim v_{\mathrm{i}}$ is not a useful ordering either, since it leads to contradictions in a general nonaxisymmetric field. ${ }^{14,15}$ Here, $\rho_{*}=\rho / L$ where $\rho$ is the ion gyroradius and $L$ is a typical macroscopic scale length. Second, if the $\boldsymbol{v}_{E}$ poloidal precession term is included in a radially local, time-independent kinetic equation for $\delta f$ (the departure of the distribution function from a Maxwellian), unphysical constraints are placed on the distribution function, as we will prove in Sec. III by considering appropriate moments of the kinetic equation. These constraints only appear when $E_{r} \neq 0$, meaning a small but nonzero $E_{r}$ is a singular perturbation of the $E_{r}=0$ case. These unphysical behaviors have been eliminated in previous codes ${ }^{4}$ by making the $a d-h o c$ replacement $1 / B^{2} \rightarrow 1 /\left\langle B^{2}\right\rangle$ (where $\langle\ldots\rangle$ denotes a flux surface average) in the $\boldsymbol{E} \times \boldsymbol{B}$ drift. At the same time, variation in the particles' energy and pitch angle associated with $E_{r}$ is neglected. These replacements and omissions are chosen so as to restore the variational form of the kinetic equation. ${ }^{5,6}$ These changes to the kinetic equation may be called the "incompressible- $\boldsymbol{E} \times \boldsymbol{B}$-drift" approximation. ${ }^{16}$ Some investigations have indicated that the incompressible- $\boldsymbol{E} \times \boldsymbol{B}$-drift approximation may be reasonably accurate for small $E_{r}$ but a poor approximation for larger $E_{r} \cdot{ }^{16,17}$ This issue of which collisionless terms to include in the kinetic equation is effectively a choice between particle trajectories, since the 
collisionless guiding center trajectories are equivalent to the characteristic curves of the drift-kinetic equation.

Another limitation of many past stellarator neoclassical calculations is that they are often performed with simplified models for collisions. The linearized Fokker-Planck collision operator-the most accurate linear operator available-has been implemented in a variety of tokamak neoclassical codes. ${ }^{18-22}$ However, due to the numerical challenge of the extra dimension in stellarators (i.e., the lack of toroidal symmetry), many stellarator neoclassical codes retain only pitch-angle scattering collisions, so coupling in the energy dimension is eliminated. The pitch-angle scattering operator lacks the momentum conservation property of the FokkerPlanck operator, which is known to be important in many situations. $^{23}$ Several techniques have been devised and implemented $\mathrm{d}^{9,24-26}$ to effectively restore momentum conservation by post-processing the transport coefficients obtained with a pure pitch-angle scattering operator, but these methods will not exactly reproduce calculations with the full linearized Fokker-Planck operator. The NEO-2 code has implemented the full linearized Fokker-Planck operator for stellarator geometry, ${ }^{10}$ but using a field-line-tracing method, which makes it difficult to add the important effect of poloidal $\boldsymbol{E} \times \boldsymbol{B}$ precession.

Here, we describe a new stellarator neoclassical code SFINCS (the Stellarator Fokker-Planck Iterative Neoclassical Conservative Solver) that can be used to explore the aforementioned issues, comparing various models for effective particle trajectories and collisions. Although we use the terminology of "effective trajectories," the code uses continuum rather than Monte Carlo algorithms. The code solves the 4D drift-kinetic equation for the distribution function, retaining coupling in 2 spatial independent variables (toroidal and poloidal angle) and 2 velocity independent variables (speed and pitch angle), but neglecting radial coupling. (For comparison, DKES ${ }^{5,6}$ is $3 \mathrm{D}$ since energy coupling is neglected, while FORTEC-3D (Refs. 11 and 12) is 5D since radial coupling is retained.) General nonaxisymmetric nested flux surface geometry is allowed, one or more species may be included, and several models for collisions are available, including the full inter-species linearized Fokker-Planck operator. The incompressible- $\boldsymbol{E} \times \boldsymbol{B}$-drift trajectories are implemented, as are several other options for trajectories that include the true $\boldsymbol{E} \times \boldsymbol{B}$ drift. As we shall demonstrate, retaining the true form of the $\boldsymbol{E} \times \boldsymbol{B}$ drift comes at a cost, requiring sources/sinks in the kinetic equation in order for the solutions to be well behaved. While all of the various options for the particle trajectories have disadvantages, SFINCS allows the options to be compared. As we will show in several calculations for the LHD and W7-X stellarators, in many experimentally relevant cases, the transport matrix elements are nearly identical for the various choices of particle trajectories. However, differences between the trajectory models emerge when the radial electric field grows comparable to the "resonant" value.

In Sec. II, we motivate the form of the kinetic equation solved by SFINCS, and detail the three models for particle trajectories that will be compared. For several of the particle trajectory models, additional sources/sinks and constraints must be included in the system of equations for the equations to be well posed and for the solutions to be well behaved. These issues are explored in Sec. III. In Sec. IV, we discuss some observations regarding momentum conservation and demonstrate that the electric field terms in the kinetic description correspond to a component of gyroviscosity in a fluid description only for the most accurate trajectory model. Details of the numerical implementation are given in Sec. V. Some of the numerical results presented are given in terms of a transport matrix, which is defined in Sec. VI. The numerical results are presented in Secs. VII and VIII in which we discuss the transport matrix elements for the geometries of the LHD and W7-X stellarators, comparing a variety of assumptions about the particle trajectories and collision operator. In Sec. IX, we discuss the results and conclude.

\section{KINETIC EQUATIONS}

We begin with the drift-kinetic Eq. (19) of Ref. 27. The standard drift ordering is applied at first: $\rho_{*_{a}} \ll 1$, where $\rho_{*_{a}}=\rho_{a} / L, \boldsymbol{v}_{E} / v_{a} \sim \rho_{* a}, \partial / \partial t \sim \rho_{* a}^{2} v_{a} / L$, and $\nu_{a} \sim v_{a} / L$. Here, $v_{a}=\sqrt{2 T_{a} / m_{a}}$ is the thermal speed of species $a, T_{a}$ is the temperature, $m_{a}$ is the mass, $\rho_{a}=v_{a} m_{a} c /\left(Z_{a} e B\right)$ is the gyroradius, $Z_{a}$ is the species charge in units of the proton charge $e, c$ is the speed of light, $L$ is a typical scale length, and $\nu_{a}$ is a collision frequency. No expansion in mass ratios or charges is made. We expand the distribution function as $f_{a}=f_{a 0}+f_{a 1}+\ldots$. The leading order distribution function $f_{a 0}$ is taken to be a Maxwellian that is constant on flux surfaces when expressed in terms of total energy $W_{a}=v^{2} / 2$ $+Z_{a} e \Phi / m_{a}$ :

$$
f_{a 0}=\eta_{a}(\psi)\left[\frac{m_{a}}{2 \pi T_{a}(\psi)}\right]^{3 / 2} \exp \left(-\frac{m_{a} W_{a}}{T_{a}(\psi)}\right) .
$$

Here, $\Phi$ is the electrostatic potential and $v$ is the speed. The mean flow of this Maxwellian is taken to be zero since, as argued in Refs. 14 and 15, sonic flows are not permitted in a general stellarator. Taking $f_{a 1} / f_{a 0} \sim \rho_{*}$, the terms of order $\sim \rho * a(v / L) f_{a 0}$ in (19) of Ref. 27 are then

$$
\begin{aligned}
v_{\|} \boldsymbol{b} \cdot\left(\nabla f_{a 1}\right)_{W_{a}, \mu}-C_{a}= & -\left(\boldsymbol{v}_{\mathrm{m} a}+\boldsymbol{v}_{E}\right) \cdot \nabla \psi\left(\frac{\partial f_{a 0}}{\partial \psi}\right)_{W_{a}} \\
& +\frac{Z_{a} e}{m_{a} c} v_{\|} \boldsymbol{b} \cdot \frac{\partial \boldsymbol{A}}{\partial t} \frac{\partial f_{a 0}}{\partial W_{a}},
\end{aligned}
$$

where the radial magnetic drift is

$$
\begin{aligned}
\boldsymbol{v}_{\mathrm{m} a} \cdot \nabla \psi & =\frac{m_{a} c v_{\|}^{2}}{Z_{a} e B} \boldsymbol{b} \times(\boldsymbol{b} \cdot \nabla \boldsymbol{b}) \cdot \nabla \psi+\frac{m_{a} c v_{\perp}^{2}}{2 Z_{a} e B^{2}} \boldsymbol{b} \times \nabla B \cdot \nabla \psi \\
& =\frac{m_{a} c}{2 Z_{a} e B^{2}}\left(v_{\|}^{2}+\frac{v_{\perp}^{2}}{2}\right) \boldsymbol{b} \times \nabla B \cdot \nabla \psi
\end{aligned}
$$

(exactly true for any $\beta$ in a magnetic equilibrium with isotropic pressure) and the $\boldsymbol{E} \times \boldsymbol{B}$ drift is $\boldsymbol{v}_{E}=\left(c / B^{2}\right) \boldsymbol{B} \times \nabla \Phi$. Here, $\boldsymbol{b}=\boldsymbol{B} / B$ is the unit vector along the magnetic field, $v_{\|}$ and $v_{\perp}$ denote the components of velocity parallel and perpendicular to $\boldsymbol{B}, 2 \pi \psi$ is the toroidal flux, $\boldsymbol{A}$ is the magnetic 
vector potential, and $C_{a}$ is the collision term for species $a$, linearized about the Maxwellians (1). Subscripts on gradients and partial derivatives indicate the quantities held fixed, and $\mu=v_{\perp}^{2} /(2 B)$ is the magnetic moment.

Unfortunately, (2) does not contain the physics of $\boldsymbol{E} \times \boldsymbol{B}$ precession, since the characteristic curves of this equation correspond only to motion along the magnetic field lines. Consequently, important transport regimes such as the $\sqrt{\nu}$ regime cannot be obtained using (2). To retain $\boldsymbol{E} \times \boldsymbol{B}$ precession, we also keep the term $\left(\boldsymbol{v}_{E}+\boldsymbol{v}_{\mathrm{m} a}\right) \cdot \nabla f_{a 1}$ in (2), even though according to the formal ordering it should appear at next order. A similar step is made in other stellarator neoclassical calculations. ${ }^{5,6}$ The mathematical reason why this term is important at low collisionality is that it has different symmetry properties than other, possibly larger, terms in (2). For instance, it survives if a bounce average is used to annihilate the first term. (We will not bounce average the kinetic equation here, but when the collisionality is low, the solution of the full equation becomes asymptotically close to the solution of the bounce-averaged equation.)

As shown in Appendix C of Ref. 28, we may choose the gauge for the electromagnetic potentials such that

$$
-c^{-1} \boldsymbol{b} \cdot \partial \boldsymbol{A} / \partial t=\left\langle E_{\|} B\right\rangle B /\left\langle B^{2}\right\rangle
$$

on the right-hand side of (2). Here, angle brackets denote a flux surface average

$$
\langle\ldots\rangle=\frac{1}{V^{\prime}} \int_{0}^{2 \pi} d \theta \int_{0}^{2 \pi} d \zeta \frac{(\ldots)}{\boldsymbol{B} \cdot \nabla \zeta},
$$

where $V^{\prime}=\int_{0}^{2 \pi} d \theta \int_{0}^{2 \pi} d \zeta / \boldsymbol{B} \cdot \nabla \zeta, \theta$ and $\zeta$ are poloidal and toroidal magnetic angles satisfying

$$
\boldsymbol{B}=\nabla \psi \times \nabla \theta+\imath \nabla \zeta \times \nabla \psi
$$

$\imath=1 / q$ is the rotational transform, and $q$ is the safety factor. Thus, (2) becomes

$$
\begin{aligned}
& \left(v_{\|} \boldsymbol{b}+\boldsymbol{v}_{E}+\boldsymbol{v}_{\mathrm{m} a}\right) \cdot\left(\nabla f_{a 1}\right)_{W_{a}, \mu}-C_{a} \\
& \quad=-\left(\boldsymbol{v}_{\mathrm{m} a}+\boldsymbol{v}_{E}\right) \cdot \nabla \psi\left(\frac{\partial f_{a 0}}{\partial \psi}\right)_{W_{a}}+\frac{Z_{a} e}{T_{a}} v_{\|} \frac{B\left\langle E_{\|} B\right\rangle}{\left\langle B^{2}\right\rangle} f_{a 0} .
\end{aligned}
$$

Even if the radial electric field is considered an input, this form of the kinetic equation remains nonlinear in the unknowns since the $\nabla f_{a 1}$ term depends on the variation of $\Phi$ on a flux surface, and this variation is an unknown like $f_{a 1}$.

To make the problem linear, we make use of the fact that the electrostatic potential is nearly a flux function. We define $\Phi_{0}=\langle\Phi\rangle$ and $\Phi_{1}=\Phi-\Phi_{0}$. We assume $\Phi_{1} \ll \Phi_{0}$, and we will show shortly that this assumption is self-consistent. Since $e \Phi_{0} / T_{a} \sim 1$ in the drift ordering, then $e \Phi_{1} / T_{a} \ll 1$. We do not expand in the ion charge $Z_{a}$. Equation (1) then gives $f_{a 0} \approx F_{a}\left[1-Z_{a} e \Phi_{1} / T_{a}\right]$ where

$$
F_{a}=n_{a}(\psi)\left[\frac{m_{a}}{2 \pi T_{a}(\psi)}\right]^{3 / 2} \exp \left(-\frac{m_{a} v^{2}}{2 T_{a}(\psi)}\right)
$$

and $n_{a}=\eta_{a} \exp \left(-Z_{a} e \Phi_{0} / T_{a}\right)$ is the leading order density. We define the leading-order total energy $W_{a 0}=v^{2} / 2$
$+Z_{a} e \Phi_{0} / m_{a}$, and leading-order $\boldsymbol{E} \times \boldsymbol{B}$ drift $\boldsymbol{v}_{E 0}=\left(c / B^{2}\right)$ $\left(d \Phi_{0} / d \psi\right) \boldsymbol{B} \times \nabla \psi$. As the relative differences between $f_{a 0}$ and $F_{a}$, between $W_{a}$ and $W_{a 0}$, and between $\boldsymbol{v}_{E}$ and $\boldsymbol{v}_{E 0}$ are all small, we may replace the former quantities with the latter ones in (7). At the same time, we note

$$
\frac{\boldsymbol{v}_{E} \cdot \nabla \psi}{\boldsymbol{v}_{\mathrm{m} a} \cdot \nabla \psi} \sim \frac{1}{\epsilon} \frac{Z_{a} e \Phi_{1}}{T_{a}}
$$

where $\epsilon$ is the relative variation of $B$ on a flux surface, and taking the ratio (9) to be small, the $\boldsymbol{v}_{E} \cdot \nabla \psi$ term in (7) may be neglected. Thus, we obtain

$$
\begin{aligned}
& \left(v_{\|} \boldsymbol{b}+\boldsymbol{v}_{E 0}+\boldsymbol{v}_{\mathrm{m} a}\right) \cdot\left(\nabla f_{a 1}\right)_{W_{a 0}, \mu}-C_{a} \\
& =-\left(\boldsymbol{v}_{\mathrm{m} a} \cdot \nabla \psi\right)\left(\frac{\partial F_{a}}{\partial \psi}\right)_{W_{a 0}}+\frac{Z_{a} e}{T_{a}} v_{\|} \frac{B\left\langle E_{\|} B\right\rangle}{\left\langle B^{2}\right\rangle} F_{a},
\end{aligned}
$$

where $C_{a}$ is now the collision operator linearized about $F_{a}$ rather than $f_{a 0}$,

$$
\left(\frac{\partial F_{a}}{\partial \psi}\right)_{W_{a 0}}=\left[\frac{1}{p_{a}} \frac{d p_{a}}{d \psi}+\frac{Z_{a} e}{T_{a}} \frac{d \Phi_{0}}{d \psi}+\left(x_{a}^{2}-\frac{5}{2}\right) \frac{1}{T_{a}} \frac{d T_{a}}{d \psi}\right] F_{a},
$$

and $x_{a}=v / v_{a}$. If $F_{a}$ and $\Phi_{0}$ are considered known, then (10) is now linear in the unknowns $f_{a 1}$, and $\Phi_{1}$ has decoupled from the kinetic equations.

We note that in some circumstances, the ratio (9) may not be small, ${ }^{3}$ particularly for impurities ${ }^{13}$ with $Z_{a} \gg 1$. However, treating the ratio (9) as finite leads to a kinetic equation that is nonlinear in the unknowns. We neglect these nonlinear effects of $\Phi_{1}$ in the present linear study, but such effects will be important to examine in future work.

For numerical computations, it is convenient to use coordinates for which the ranges of allowed values are independent of the other coordinates. As $W_{a 0}$ and $\mu$ do not have this property, it is convenient to switch to coordinates $x_{a}$ and $\xi=v_{\|} / v$. Carrying out this change of variables on the first term of (10), we find

$$
\begin{aligned}
\dot{\boldsymbol{r}} \cdot\left(\nabla f_{a 1}\right)_{W_{a 0}, \mu}= & \dot{\boldsymbol{r}} \cdot\left(\nabla f_{a 1}\right)_{x_{a}, \xi}+\dot{x}_{a}\left(\frac{\partial f_{a 1}}{\partial x_{a}}\right)_{\boldsymbol{r}, \xi} \\
& +\dot{\xi}_{a}\left(\frac{\partial f_{a 1}}{\partial \xi}\right)_{\boldsymbol{r}, x_{a}},
\end{aligned}
$$

where $\boldsymbol{r}$ denotes the position vector,

$$
\begin{gathered}
\dot{\boldsymbol{r}}=v_{\|} \boldsymbol{b}+\boldsymbol{v}_{E 0}+\boldsymbol{v}_{\mathrm{m} a} \\
\dot{x}_{a}=\left(\boldsymbol{v}_{\mathrm{m} a} \cdot \nabla \psi\right)\left(-\frac{x_{a}}{2 T_{a}} \frac{d T_{a}}{d \psi}-\frac{Z_{a} e}{2 T_{a} x_{a}} \frac{d \Phi_{0}}{d \psi}\right),
\end{gathered}
$$

and

$$
\begin{aligned}
\dot{\xi}_{a}= & -\frac{1-\xi^{2}}{2 B \xi} v_{\|} \boldsymbol{b} \cdot \nabla B+\xi\left(1-\xi^{2}\right) \frac{c}{2 B^{3}} \frac{d \Phi_{0}}{d \psi} \boldsymbol{B} \times \nabla \psi \cdot \nabla B \\
& -\frac{1-\xi^{2}}{2 B \xi} \boldsymbol{v}_{\mathrm{m} a} \cdot \nabla B .
\end{aligned}
$$


For the rest of this work, we will neglect the $\boldsymbol{v}_{\mathrm{m} a}$ term in (13), the $d T_{a} / d \psi$ term in (14), and the $\boldsymbol{v}_{\mathrm{m} a} \cdot \nabla B$ term in (15), for several reasons. First, if the $v_{\text {ma }}$ term in (13) was retained, we would need to solve a $5 \mathrm{D}$ rather than $4 \mathrm{D}$ problem due to the radial coupling (i.e., $\psi$ appearing as a derivative rather than merely as a parameter). Second, once radial coupling is dropped, we must also drop the $d T_{a} / d \psi$ term in (14) and the $v_{\mathrm{m} a} \cdot \nabla B$ term in (15) in order for $\mu$ to be conserved. Third, dropping these terms conveniently eliminates all dependence of the transport matrix (defined in Sec. VI) on $d T_{a} / d \psi, d B / d \psi$, and $\rho_{*}$. Fourth, dropping these terms amounts to taking the limit $\rho_{*} \rightarrow 0$ (while keeping the $d \Phi_{0} / d \psi$ terms finite), and this limit is already complicated and interesting to explore without the extra complexity of finite- $\rho *$ corrections. Fifth, we wish to focus on the effects of the radial electric field. The omitted terms may be important in other situations, but here our primary interest is the treatment of the $d \Phi_{0} / d \psi$ terms. Finally, these omitted terms would significantly complicate the analysis in Sec. III in which we will examine moments of the kinetic equation.

Our kinetic equation then becomes

$$
\begin{gathered}
\dot{\boldsymbol{r}} \cdot\left(\nabla f_{a 1}\right)_{x_{a}, \xi}+\dot{x}_{a}\left(\frac{\partial f_{a 1}}{\partial x_{a}}\right)_{\boldsymbol{r}, \xi}+\dot{\xi}_{a}\left(\frac{\partial f_{a 1}}{\partial \xi}\right)_{\boldsymbol{r}, x_{a}}-C_{a} \\
=-\left(\boldsymbol{v}_{\mathrm{m} a} \cdot \nabla \psi\right)\left(\frac{\partial F_{a}}{\partial \psi}\right)_{W_{a}}+\frac{Z_{a} e}{T_{a}} v_{\|} \frac{B\left\langle E_{\|} B\right\rangle}{\left\langle B^{2}\right\rangle} F_{a},
\end{gathered}
$$

where the effective particle trajectory equations are

$$
\begin{aligned}
\dot{\boldsymbol{r}}= & v_{\|} \boldsymbol{b}+\frac{c}{B^{2}} \frac{d \Phi_{0}}{d \psi} \boldsymbol{B} \times \nabla \psi, \\
\dot{x}_{a}= & -\left(\boldsymbol{v}_{\mathrm{m} a} \cdot \nabla \psi\right) \frac{Z_{a} e}{2 T_{a} x_{a}} \frac{d \Phi_{0}}{d \psi}, \\
\dot{\xi}_{a}= & -\frac{1-\xi^{2}}{2 B \xi} v_{\|} \boldsymbol{b} \cdot \nabla B \\
& +\xi\left(1-\xi^{2}\right) \frac{c}{2 B^{3}} \frac{d \Phi_{0}}{d \psi} \boldsymbol{B} \times \nabla \psi \cdot \nabla B .
\end{aligned}
$$

We will refer to (17) as the "full trajectories."

The $d \Phi_{0} / d \psi$ terms in $\dot{x}_{a}$ and $\dot{\xi}_{a}$ may be interpreted as a finite orbit width effect. As a particle drifts radially, it experiences a varying electrostatic potential (even if the potential is a flux function.) Thus, the potential energy of the particle changes, so to maintain a constant total energy, the kinetic energy must change at an equal and opposite rate, giving rise to the $d \Phi / d \psi$ term in $\dot{x}_{a}$. Then to conserve $\mu$ while $v$ changes, $\xi$ must also change appropriately, giving rise to the $d \Phi_{0} / d \psi$ term in $\dot{\xi}_{a}$. Without these $d \Phi_{0} / d \psi$ terms in $\dot{x}_{a}$ and $\dot{\xi}_{a}, \mu$ will not be conserved, whereas you can verify that $\mu$ is indeed conserved by (17). Note that the $d \Phi_{0} / d \psi$ term in $\dot{\boldsymbol{r}}$ is the same order in the $\rho_{*}$ expansion as the $d \Phi_{0} / d \psi$ terms in $\dot{x}_{a}$ and $\dot{\xi}_{a}$, suggesting that if the former term is retained, the latter terms should be retained as well.

A large number of stellarator neoclassical codes ${ }^{4-6}$ effectively solve (16) with the alternative trajectory equations

$$
\begin{aligned}
\dot{\boldsymbol{r}} & =v_{\|} \boldsymbol{b}+\frac{c}{\left\langle B^{2}\right\rangle} \frac{d \Phi_{0}}{d \psi} \boldsymbol{B} \times \nabla \psi, \\
\dot{x}_{a} & =0, \\
\dot{\xi}_{a} & =-\frac{1-\xi^{2}}{2 B \xi} v_{\|} \boldsymbol{b} \cdot \nabla B .
\end{aligned}
$$

We refer to these equations as the "DKES trajectories," in light of their use in the widely applied code DKES. ${ }^{5,6}$ These trajectories differ from (17) both in the neglect of the $d \Phi_{0} / d \psi$ terms in $\dot{x}_{a}$ and $\dot{\xi}_{a}$, and in the replacement $B^{2} \rightarrow\left\langle B^{2}\right\rangle$ in $\dot{\boldsymbol{r}}$. The motivation for approximating the $\boldsymbol{E} \times \boldsymbol{B}$ drift in this matter will be clarified in Sec. III. As shown in Refs. 17 and 29, in a symmetric magnetic field, the model (18) possesses a conserved quantity, which is equal to $\mu$ when $d \Phi_{0} / d \psi=0$ but which differs from $\mu$ when $d \Phi_{0} / d \psi \neq 0$.

For comparison, we will also consider the following set of trajectory equations:

$$
\begin{aligned}
\dot{\boldsymbol{r}} & =v_{\|} \boldsymbol{b}+\frac{c}{B^{2}} \frac{d \Phi_{0}}{d \psi} \boldsymbol{B} \times \nabla \psi, \\
\dot{x}_{a} & =0, \\
\dot{\xi}_{a} & =-\frac{1-\xi^{2}}{2 B \xi} v_{\|} \boldsymbol{b} \cdot \nabla B,
\end{aligned}
$$

which will be referred to as the "partial trajectories." Equations (19) represent an intermediate step between (17) and (18), in that (19) includes the correct $\boldsymbol{E} \times \boldsymbol{B}$ drift, but not the $d \Phi_{0} / d \psi$ terms in $\dot{x}_{a}$ and $\dot{\xi}_{a}$ required to conserve $\mu$.

Note that for both the DKES and full trajectories, the left-hand side of the kinetic Eq. (16) can be written in the conservative form

$$
\frac{1}{J}\left[\nabla \cdot\left(J \dot{\boldsymbol{r}}_{a} f_{a 1}\right)+\frac{\partial}{\partial \xi}\left(J \dot{\xi}_{a} f_{a 1}\right)+\frac{\partial}{\partial x_{a}}\left(J \dot{x}_{a} f_{a 1}\right)\right]-C_{a},
$$

where $J=x_{a}^{2}$ is the Jacobian of the transformation between Cartesian velocity coordinates and the coordinates $x_{a}, \xi$, and gyrophase. However, for the partial trajectories, the left-hand side of (16) is not equivalent to (20).

For all three trajectory models, the quasineutrality equation is effectively decoupled from the kinetic Eq. (16). At leading order, quasineutrality implies $\sum_{a} Z_{a} n_{a}=0$. At next order, noting that both $f_{a 0}$ and $f_{a 1}$ contribute to density variation on a flux surface,

$$
\sum_{a}\left(-\frac{Z_{a}^{2} e \Phi_{1}}{T_{a}} n_{a}+Z_{a} \int d^{3} v f_{a 1}\right)=0 .
$$

This equation may be solved for $\Phi_{1}$, giving the variation of the potential on a flux surface. It follows that $e \Phi_{1} / T_{a}$ $\sim f_{a 1} / f_{a 0} \sim \rho_{* a}$, so our earlier assumption that $e \Phi_{1} / T_{a} \ll 1$ is self-consistent.

Several choices can be made for the collision operator. The most accurate linear option is the Fokker-Planck operator $^{30,31}$ linearized about the Maxwellians: $C_{a}=\sum_{b} C_{a b}^{\ell}$, where $C_{a b}^{\ell}=C_{a b}\left\{f_{a 1}, F_{b}\right\}+C_{a b}\left\{F_{a}, f_{b 1}\right\}$ and $C_{a b}$ is the full bilinear Fokker-Planck operator between species $a$ and $b$. This linearized operator may be written in many forms; for 
numerical implementation, we find it convenient to use the form detailed in Eqs. (14)-(16) of Ref. 22.

A simpler option used in many codes is the pitch-angle scattering operator. ${ }^{4}$ This operator lacks several properties of the linearized Fokker-Planck operator, such as the momentum conservation property $\int d^{3} v v_{\|} C_{a a}^{\ell}=0$. Several more accurate approximate operators have been used in the literature. One such operator we will consider later consists of the pitch-angle scattering operator plus an ad-hoc momentumrestoring term, given for the case of self-collisions by Eq. (3.69) in Ref. 31.

\section{PARTICLE AND ENERGY MOMENT EQUATIONS, CONSERVATION PROPERTIES, AND SOURCES}

If one attempts to solve the kinetic Eq. (16) numerically using either the full or partial trajectories and $E_{r} \neq 0$, unphysical results will be obtained, with the numerical solution not converging as resolution parameters are increased. We now explore the reason for this behavior. We will then describe a modified form of the kinetic equation, which robustly produces more sensible results. The issues discussed in this section are related to moment equations for mass and energy; momentum has a different status and will be examined in Sec. IV.

Consider the result of applying the operation

$$
\left\langle\int d^{3} v(\ldots)\right\rangle
$$

to the kinetic Eq. (16) for each of the trajectory models (17)-(19). This operation annihilates the streaming and mirror terms, the collision operator, and the inhomogeneous drive terms. The operation (22) effectively produces a fluxsurface-averaged mass conservation equation for each model. For the full trajectories and DKES trajectories, the $d \Phi_{0} / d \psi$ terms are also annihilated by (22), so the resulting mass conservation equation is just $0=0$. However, for the partial trajectories, the $d \Phi_{0} / d \psi$ term $\left(\boldsymbol{v}_{E 0} \cdot \nabla f_{a 1}\right)$ is not annihilated by (22), leaving

$$
c\left\langle\frac{1}{B^{2}} \boldsymbol{B} \times \nabla \psi \cdot \nabla \int d^{3} v f_{a 1}\right\rangle \frac{d \Phi_{0}}{d \psi}=0 .
$$

Thus, a nonzero $d \Phi_{0} / d \psi$ gives a singular perturbation to the $d \Phi_{0} / d \psi=0$ limit in this partial trajectory model: the $d \Phi_{0} / d \psi=0$ solution for $f_{a 1}$ need not satisfy $\left\langle\left(1 / B^{2}\right) \boldsymbol{B} \times \nabla \psi\right.$ $\left.\cdot \nabla \int d^{3} v f_{a 1}\right\rangle=0$, so $f_{a 1}$ must change dramatically as $E_{r}$ is raised from 0 to a small nonzero value, a behavior which is unphysical. When $d \Phi_{0} / d \psi \neq 0$, (23) constrains $f_{a 1}$ in an unphysical manner, for there is no analogue to (23) in the more accurate averaged fluid mass conservation equation $0=\left\langle\partial N_{a} / \partial t+\nabla \cdot\left(N_{a} \boldsymbol{V}_{a}\right)\right\rangle$ (i.e., the moment of the full Fokker-Planck equation with no expansion in $\rho_{*}$ or other parameters), where $N_{a}$ and $\boldsymbol{V}_{a}$ are the full fluid density and velocity. The unphysical nature of (23) can also be seen from the fact that when the $d \Phi_{0} / d \psi$ terms in $\dot{x}_{a}$ and $\dot{\xi}_{a}$ are retained in the more accurate trajectories (17), these terms precisely cancel (23).

Similarly, we can obtain an averaged energy conservation equation for each trajectory model by applying the operation

$$
\sum_{a}\left\langle\int d^{3} v \frac{m_{a} v^{2}}{2}(\ldots)\right\rangle
$$

to (16). Again, the result is $0=0$ for the DKES trajectories. However, this time both the full and partial trajectory models give nonzero results: the partial trajectories give

$$
c \sum_{a}\left\langle\frac{1}{B^{2}} \boldsymbol{B} \times \nabla \psi \cdot \nabla \int d^{3} v \frac{m_{a} v^{2}}{2} f_{a 1}\right\rangle \frac{d \Phi_{0}}{d \psi}=0
$$

and the full trajectories give

$$
-c \sum_{a}\left\langle\frac{1}{B^{2}} \boldsymbol{B} \times \nabla \psi \cdot \nabla \int d^{3} v \frac{m_{a} v^{2}}{2} \frac{\left(1+\xi^{2}\right)}{2} f_{a 1}\right\rangle \frac{d \Phi_{0}}{d \psi}=0 .
$$

The quantity multiplying $d \Phi_{0} / d \psi$ in (26) is proportional to the radial current $\sum_{a} Z_{a}\left\langle\int d^{3} v f_{a 1} \boldsymbol{v}_{\mathrm{m} a} \cdot \nabla \psi\right\rangle$, so it vanishes naturally when $E_{r}$ is at the ambipolar value. However, as the radial current would usually not be zero when $E_{r}=0$, (26) again implies a small nonzero $E_{r}$ would be a singular perturbation of the $E_{r}=0$ limit.

One motivation for use of the DKES trajectory model is now apparent: it is the only model (of the three considered here) that avoids the imposition of one or more unphysical constraints on the distribution function when $d \Phi_{0} / d \psi \neq 0$, constraints which cause an $E_{r} \neq 0$ calculation to be a singular perturbation of an $E_{r}=0$ calculation.

The aforementioned problems with the partial and full trajectory models may be eliminated in the following manner. The kinetic equation becomes well behaved if we introduce particle and heat sources

$$
\begin{aligned}
S_{a}(\psi, v)= & S_{a \mathrm{p}}(\psi) F_{a}(\psi, v)\left[x_{a}^{2}-\frac{5}{2}\right] \\
& +S_{a \mathrm{~h}}(\psi) F_{a}(\psi, v)\left[x_{a}^{2}-\frac{3}{2}\right],
\end{aligned}
$$

where $S_{a \mathrm{p}}$ and $S_{a \mathrm{~h}}$ are considered to be unknowns. (The factors involving $x_{a}^{2}$ in (27) are chosen so $S_{a \mathrm{p}}$ provides a particle source but no heat source, while $S_{a \text { h }}$ provides a heat source but no particle source.) As these two new unknowns are now included in the system of equations on each flux surface, we must supply an equal number of additional constraints. The constraints we supply are $\left\langle\int d^{3} v f_{a 1}\right\rangle=0$ and $\left\langle\int d^{3} v v^{2} f_{a 1}\right\rangle=0$, the sensible requirements that all the flux-surface-averaged density and pressure reside in $F_{a}$ rather than $f_{a 1}$. When $S_{a}$ is included in the kinetic equation, new terms proportional to $S_{a \mathrm{p}}$ and/or $S_{a \mathrm{~h}}$ now appear in the mass and energy conservation equations such as (23)-(26). These conservation equations imply that when $d \Phi_{0} / d \psi=0, S_{a \mathrm{p}}$ and $S_{a \mathrm{~h}}$ must vanish. However, now when $d \Phi_{0} / d \psi$ is increased from 0 to a small finite number, the sources can turn on to satisfy (23)-(26), eliminating the singular perturbation in $f_{a 1}$. We find that numerical results are then well behaved, converging appropriately as numerical resolution parameters are increased, and smoothly going to the $E_{r}=0$ results as $E_{r}$ is decreased.

We do not claim that the method proposed here is an ideal solution: the sources (27) are ad-hoc and are not 
derived rigorously. However, by the techniques proposed here, we can at least compare the three different trajectory models, and for most experimentally relevant values of $E_{r}$, we will show that the three models give nearly identical results. And as already mentioned, the source terms for the full trajectory model are both zero when the radial electric field equals the value required for ambipolarity, so for this model, the source terms are really a numerical expedient that do not affect the transport computations in the end.

This system of sources and constraints solves not only the problem described above when $E_{r} \neq 0$ but also a different problem that remains even when $E_{r}=0$ and/or when the DKES trajectories are used: the kinetic equation has a null space. If the conditions $\left\langle\int d^{3} v f_{a 1}\right\rangle=0$ and $\left\langle\int d^{3} v v^{2} f_{a 1}\right\rangle=0$ were not imposed, any linear combination of $F_{a}$ and $F_{a} v^{2}$ could be added to one solution of the kinetic equation to obtain another solution. Upon discretization, one would obtain a non-invertible (or at least very poorly conditioned) linear system, but the imposition of these two extra constraints makes the system of equations invertible.

Such is the case when the full linearized Fokker-Planck collision operator is used, but the situation is different when either the pitch-angle scattering operator or momentum-conserving model operator are used instead, for then the kinetic equation has a larger null space: any function of $v$ is then a homogeneous solution of the kinetic equation. As the dimension of the null space is then equal to $N_{x}$ (the number of grid points in $x_{a}$ ) rather than 2, it takes $N_{x}$ rather than 2 constraint equations to eliminate the null space for these collision operators. We choose these $N_{x}$ constraints to be $\left\langle\int_{-1}^{1} d \xi f_{a 1}\right\rangle=0$ (imposed at each grid point in $x_{a}$ ). To keep the linear system square, we must then have $N_{x}$ rather than 2 unknowns related to the sources. This is accomplished by letting the source be a general function of $x_{a}$ instead of (27) when either the pitch-angle scattering or momentum-conserving model collision operator are used. This alternative system of $N_{x}$ sources and constraints is an equally reasonable solution to the earlier conservation problem.

To summarize, the sources and extra constraint equations serve two independent purposes. First, when $E_{r} \neq 0$, the sources are needed to eliminate the conservation problems, and the extra constraints then keep the linear system square (number of equations $=$ number of unknowns) upon discretization. Second, even when $E_{r}=0$, and even for the DKES trajectories in which sources are not required, the constraints are needed to eliminate the null space in the kinetic equation, and the source terms are a convenient way to keep the linear system square upon discretization. The first problem can be solved with either the source (27) or a general speed-dependent source $S_{a}\left(x_{a}\right)$. However, to solve the second problem, the number of constraints should match the dimensionality of the null space. For this reason, we apply the source (27) with 2 constraints when the Fokker-Planck operator is used, while we apply the general speed-dependent source $S_{a}\left(x_{a}\right)$ with $N_{x}$ constraints when either of the other two collision operators is used.

\section{MOMENTUM MOMENT EQUATIONS}

Parallel momentum has a different status to density and energy, in that density and energy are conserved by the collisionless motion while parallel momentum is not, due to the mirror force. (For example, considering the case of a single ion species with $d \Phi_{0} / d \psi=0, F_{i}$ and $v^{2} F_{i}$ are homogeneous solutions to the kinetic equation, whereas $v_{\|} F_{i}$ is not.) A consequence is that there does not appear to be a false constraint for $E_{r} \neq 0$ arising from the $\left\langle\int d^{3} v v_{\|}(\ldots)\right\rangle$ moment of the various forms of the kinetic equation, i.e., there is no analogue to (23), (25), or (26) for momentum. When the momentum moment of the various forms of the drift-kinetic equation is taken, even if a factor of $B$ or $1 / B$ is included in the flux surface average, a collisionless term remains that is not proportional to $d \Phi_{0} / d \psi$. Consequently, for all the trajectory models, $d \Phi_{0} / d \psi=0$ is a well behaved rather than singular limit of the momentum moment equation.

Nonetheless, it is interesting to compare the $\int d^{3} v m_{a} v_{\|}$ (...) moment equations for each drift-kinetic trajectory model to the full parallel momentum fluid equation. This later equation, the moment of the full Fokker-Planck equation, is

$$
0=-\boldsymbol{b} \cdot\left(\nabla \cdot \Pi_{a}\right)+Z_{a} e n_{a} E_{\|}+F_{a \|},
$$

where $\boldsymbol{\Pi}_{\boldsymbol{a}}=m_{a} \int d^{3} v f_{a} \boldsymbol{v} \boldsymbol{v}$ is the stress tensor and $F_{a \|}$ is the parallel component of friction. First, consider the case of no radial electric field. Recalling $f_{a}=F_{a}(\psi)\left[1-Z_{a} e \Phi_{1} / T_{a}\right]$ $+f_{a 1}$, the stress tensor is given to the accuracy needed by $\boldsymbol{\Pi}_{a} \approx p_{a}(\psi)\left[1-Z_{a} e \Phi_{1} / T_{a}\right] \boldsymbol{I}+\boldsymbol{\Pi}_{a 1} \quad$ where $\quad \boldsymbol{\Pi}_{a 1}=p_{a 1 \perp} \boldsymbol{I}$ $+\left(p_{a 1 \|}-p_{a 1 \perp}\right) \boldsymbol{b} \boldsymbol{b}, \quad p_{a 1 \|}=m_{a} \int d^{3} v f_{a 1} v_{\|}^{2}, \quad$ and $\quad p_{a 1 \perp}=m_{a}$ $\int d^{3} v f_{a 1} v_{\perp}^{2} / 2$. Notice the $\int d^{3} v m_{a} v_{\|}(\ldots)$ moment of the streaming and mirror terms in (16)-(17) is

$$
\begin{aligned}
& \int d^{3} v m_{a} v_{\|}\left[v_{\|} \boldsymbol{b} \cdot \nabla f_{a 1}-\frac{1-\xi^{2}}{2 B} v(\boldsymbol{b} \cdot \nabla B) \frac{\partial f_{a 1}}{\partial \xi}\right] \\
& =\boldsymbol{b} \cdot \nabla p_{a 1 \|}+\frac{p_{a 1 \perp}-p_{a 1 \|}}{B} \boldsymbol{b} \cdot \nabla B \\
& =\boldsymbol{b} \cdot\left(\nabla \cdot \boldsymbol{\Pi}_{a 1}\right) .
\end{aligned}
$$

Using this result with (4), the $m_{a} v_{\|}$moment of the driftkinetic Eqs. (16) and (17) matches the full fluid parallel momentum Eq. (28) at least when $E_{r}=0$.

Now consider how the situation changes when a radial electric field is introduced. We first compute the change to the fluid parallel momentum equation caused by a new contribution to the viscosity. Examining the $\left(\boldsymbol{E}+c^{-1} \boldsymbol{v} \times \boldsymbol{B}\right)$. $\nabla_{v} f_{a}$ terms in the full Fokker-Planck equation and integrating in gyrophase, one sees the gyrophase-dependent part of the distribution function $\tilde{f}_{a}$ will include the following terms proportional to the electric field:

$$
\tilde{f}_{a E}=\frac{c}{B} \boldsymbol{v} \cdot \boldsymbol{b} \times \boldsymbol{E}\left[\frac{1}{v} \frac{\partial \bar{f}_{a}}{\partial v}-\frac{\xi}{v^{2}} \frac{\partial \bar{f}_{a}}{\partial \xi}\right]
$$

as reflected (using different independent variables) in Eq. (17) of Ref. 27 and Eq. (6) of Ref. 32. Here, $\bar{f}_{a}$ is the gyrophase-independent part of the distribution function. The associated contribution $\boldsymbol{\Pi}_{a E}=m_{a} \int d^{3} v \tilde{f}_{a E} \boldsymbol{v} \boldsymbol{v}$ to the pressure tensor is calculated in Eqs. (27)-(36) of Ref. 32, with the result 


$$
\boldsymbol{\Pi}_{a E}=m_{a} n_{a} V_{a \|}\left(\boldsymbol{b} \boldsymbol{v}_{E}+\boldsymbol{v}_{E} \boldsymbol{b}\right)
$$

where $n_{a} V_{a \|}=\int d^{3} v f_{a} v_{\|}$. Note that this contribution to the stress tensor is a part of the gyroviscosity and is offdiagonal. Using $\boldsymbol{E} \approx-\nabla \Phi_{0}(\psi)$ and $\boldsymbol{B} \cdot\{\nabla \cdot[\boldsymbol{B} \boldsymbol{B} \times \nabla \psi$ $+(\boldsymbol{B} \times \nabla \psi) \boldsymbol{B}]\}=2 B^{2} \nabla \psi \cdot \nabla \times \boldsymbol{B}=0$, we then find the contribution to the parallel momentum Eq. (28) from (30)-(31) is

$$
\boldsymbol{b} \cdot\left(\nabla \cdot \boldsymbol{\Pi}_{a E}\right)=c m_{a} B\left(d \Phi_{0} / d \psi\right) \boldsymbol{B} \times \nabla \psi \cdot \nabla\left(n_{a} V_{a \|} / B^{3}\right) .
$$

For comparison, let us consider the $\int d^{3} v m_{a} v_{\|}(\ldots)$ moment of the radial electric field terms in the drift-kinetic equation for various trajectory models, to see if the results agree with (32). For the full trajectories, the moment of the $d \Phi_{0} / d \psi$ terms in (16) and (17) is

$$
\begin{aligned}
& \int d^{3} v m_{a} v_{\|}\left[\frac{c}{B^{2}} \frac{d \Phi_{0}}{d \psi} \boldsymbol{B} \times \nabla \psi \cdot \nabla f_{a 1}-\left(\boldsymbol{v}_{\mathrm{m} a} \cdot \nabla \psi\right)\right. \\
& \times \frac{Z_{a} e}{2 T_{a} x_{a}} \frac{d \Phi_{0}}{d \psi} \frac{\partial f_{a 1}}{\partial x_{a}}+\xi\left(1-\xi^{2}\right) \frac{c}{2 B^{3}} \frac{d \Phi_{0}}{d \psi} \\
& \left.\times(\boldsymbol{B} \times \nabla \psi \cdot \nabla B) \frac{\partial f_{a}}{\partial \xi}\right] \\
& =c m_{a} B\left(d \Phi_{0} / d \psi\right) \boldsymbol{B} \times \nabla \psi \cdot \nabla\left(n_{a} V_{a \|} / B^{3}\right),
\end{aligned}
$$

obtained by integrating by parts in $x_{a}$ and $\xi$. Thus, the fulltrajectory model agrees with the full fluid parallel momentum equation: $(33)=(32)$. However, this agreement is not shared by the DKES model: the moment of the $d \Phi_{0} / d \psi$ term in (18) is

$$
\begin{gathered}
\int d^{3} v m_{a} v_{\|}\left[\frac{c}{\left\langle B^{2}\right\rangle} \frac{d \Phi_{0}}{d \psi} \boldsymbol{B} \times \nabla \psi \cdot \nabla f_{a 1}\right] \\
=\frac{c m_{a}}{\left\langle B^{2}\right\rangle} \frac{d \Phi_{0}}{d \psi} \boldsymbol{B} \times \nabla \psi \cdot \nabla\left(n_{a} V_{a \|}\right),
\end{gathered}
$$

which does not equal (32). The corresponding result for the partial trajectories, obtained by replacing $\left\langle B^{2}\right\rangle \rightarrow B^{2}$ in (34), also does not match (32). Thus, the DKES and partial trajectory models do not correctly account for the parallel viscous force as the full trajectory model does.

We close this section by noting another important difference between the trajectory models related to the parallel momentum equations. Consider the case of quasisymmetry, which is the condition that $\boldsymbol{B} \times \nabla \psi \cdot \nabla B=A(\psi) \boldsymbol{B} \cdot \nabla B$ for some flux function $A(\psi))^{33}$ It was known previously ${ }^{33}$ that when the $E_{r}$ terms are not included in the trajectories (but retained in the $\partial F_{a} / \partial \psi$ drive term in (16)), the radial neoclassical current vanishes for all values of $d \Phi_{0} / d \psi$ if and only if the flux surface is quasisymmetric. This property of quasisymmetric flux surfaces is known as intrinsic ambipolarity. Here, we show that intrinsic ambipolarity persists in quasisymmetric geometry when the $E_{r}$ terms are retained in the full trajectory drift-kinetic equation, but not for the DKES or partial trajectory kinetic equations. This result follows from the $-\sum_{a} Z_{a}\left\langle\int d^{3} v A v_{\|} / \Omega_{a}(\ldots)\right\rangle$ moment of the kinetic equations, i.e., a spatially weighted average of the parallel momentum moment. For the full trajectories, (33) vanishes in this spatial average, leaving

$$
\sum_{a} Z_{a}\left\langle\int d^{3} v f_{a 1} \boldsymbol{v}_{\mathrm{m} a} \cdot \nabla \psi\right\rangle=0,
$$

meaning there is no radial current. However, for the DKES and partial trajectory models, the spatial average does not annihilate the $d \Phi_{0} / d \psi$ term, leaving an additional term in (35) proportional to $d \Phi_{0} / d \psi$, and therefore the radial current is generally nonzero. Consequently, the full trajectory model is the only one of the models that preserves intrinsic ambipolarity in quasisymmetry for $E_{r} \neq 0$. Notice that when the full trajectory model is applied in quasisymmetry, intrinsic ambipolarity means (26) is satisfied even when $d \Phi_{0} / d \psi \neq 0$, so the net heat source vanishes for any radial electric field.

\section{NUMERICAL IMPLEMENTATION}

The SFINCS code solves the drift-kinetic Eq. (16) with (27) for any of the three trajectory models (17)-(19), for general nonaxisymmetric nested flux surface geometry, and for an arbitrary number of species. SFINCS is based on the Fokker-Planck code described in Ref. 22, generalized to allow nonaxisymmetry. SFINCS is also closely related to the radially global Fokker-Planck code for tokamaks described in Ref. 34. Briefly, the kinetic equation is discretized using finite differences with a 5-point stencil in $\theta$ and $\zeta$, using a truncated Legendre modal expansion in $\xi$, and using a spectral collocation method in $x_{a}$. The time-independent kinetic equation is solved directly (by solving a single sparse linear system), so the rate of convergence is not limited by the timescale of physical relaxation. The modifications compared to the code of Ref. 22 are the following. (1) $f_{a 1}, B$, and other geometric operators are allowed to depend on the toroidal angle $\zeta$, and the numerical grid is expanded to include this new coordinate. (2) The additional $d \Phi_{0} / d \psi$ terms in $\dot{\boldsymbol{r}}_{a}, \dot{x}_{a}$, and $\dot{\xi}_{a}$ are included. (3) The additional collision operators discussed above are included. (4) The extra constraint equations and sources are implemented as in (19) of Ref. 34. Specifically, considering first the case of a single species for simplicity, the linear system has the block structure

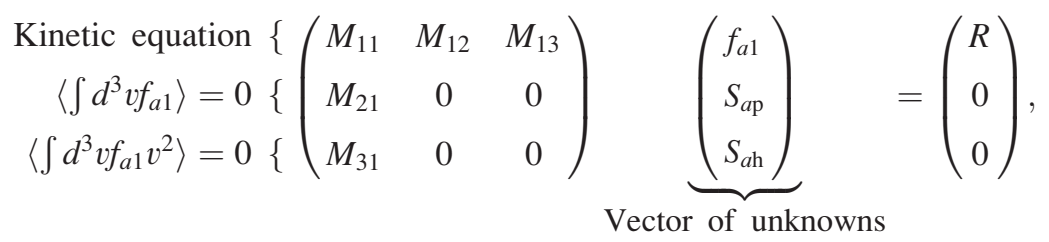


where $R$ is the inhomogeneous term (i.e., the right-hand side) from (16), and the $M$ operators are as follows: $M_{11}$ represents the operator on the left-hand side of (16), $M_{12}$ and $M_{13}$ represent the $S_{a \mathrm{p}}$ and $S_{a \mathrm{~h}}$ terms in (27), respectively, and $M_{21}$ and $M_{31}$ represent the aforementioned extra constraint equations introduced. For the case of multiple particle species, the linear system consists of blocks of the form (36) for each species, with coupling between species only through the collision operators in the $M_{11}$ blocks.

The resulting large sparse linear system is solved using the PETSc ${ }^{35,36}$ library. A preconditioned iterative Krylov solver is employed, either GMRES ${ }^{37}$ or BICGStab(1). ${ }^{38}$ An effective preconditioner is typically obtained by dropping all coupling in the $x_{a}$ coordinate, either for all Legendre modes in $\xi$, or for all but the first one or two Legendre modes. The preconditioner is $L U$-factorized directly using the SuperLU-dist ${ }^{39,40}$ package.

Note that poloidal and toroidal magnetic drifts could be included in the kinetic equation without increasing the density of the matrix, i.e., without increasing the computational expense of the method here. We do not expect any fundamental new complications to arise if poloidal and toroidal magnetic drift terms are retained. However, to include radial drifts acting on $f_{a 1}$, the number of independent variables would increase from 4 to 5 since different flux surfaces would couple. This increase in dimensionality would be numerically challenging.
The magnetic geometry is specified in Boozer coordinates $\theta$ and $\zeta$ in which

$$
\boldsymbol{B}=\beta(\psi, \theta, \zeta) \nabla \psi+I(\psi) \nabla \theta+G(\psi) \nabla \zeta .
$$

Here, $c I / 2$ is the toroidal current inside the flux surface, and $c G / 2$ is the poloidal current outside the flux surface. The geometric operators needed in the kinetic equation are then

$$
\boldsymbol{B} \cdot \nabla X=\left(l \frac{\partial X}{\partial \theta}+\frac{\partial X}{\partial \zeta}\right) \boldsymbol{B} \cdot \nabla \zeta
$$

and

$$
\boldsymbol{B} \times \nabla \psi \cdot \nabla X=\left(G \frac{\partial X}{\partial \theta}-I \frac{\partial X}{\partial \zeta}\right) \boldsymbol{B} \cdot \nabla \zeta,
$$

where $X$ can be any scalar quantity, and the inverse coordinate Jacobian is $\boldsymbol{B} \cdot \nabla \zeta=B^{2} /(G+\imath I)$. Thus, the magnetic geometry enters the kinetic equation only through the quantities $I, G, l$, and $B(\theta, \zeta)$.

\section{ION TRANSPORT MATRIX}

We will present results of the numerical calculations in terms of the transport matrix $L_{j k}$, defined as follows:

$$
\left(\begin{array}{c}
\frac{Z e(G+\imath I)}{n c T G}\left\langle\int d^{3} v f \boldsymbol{v}_{\mathrm{m}} \cdot \nabla \psi\right\rangle \\
\frac{Z e(G+\imath I)}{n c T G}\left\langle\int d^{3} v f \frac{m v^{2}}{2 T} \boldsymbol{v}_{\mathrm{m}} \cdot \nabla \psi\right\rangle \\
\frac{1}{v_{\mathrm{i}} B_{0}}\left\langle B V_{\|}\right\rangle
\end{array}\right)=\left(\begin{array}{ccc}
L_{11} & L_{12} & L_{13} \\
L_{21} & L_{22} & L_{23} \\
L_{31} & L_{32} & L_{33}
\end{array}\right)\left(\begin{array}{c}
\frac{G T c}{Z e B_{0} v_{i}}\left[\frac{1}{n} \frac{d n}{d \psi}+\frac{Z e}{T} \frac{d \Phi}{d \psi}-\frac{3}{2 T} \frac{d T}{d \psi}\right] \\
\frac{G T c}{Z e B_{0} v_{i} T} \frac{d T}{d \psi} \\
\frac{Z e}{T}(G+\imath I) \frac{\left\langle E_{\|} B\right\rangle}{\left\langle B^{2}\right\rangle}
\end{array}\right)
$$

Here, $B_{0}$ is the $(0,0)$ Fourier mode amplitude of $B(\theta, \zeta)$, and we have dropped $i$ subscripts where possible to simplify the notation. When the DKES trajectories (18) are used, it can be shown that $L_{j k}$ is symmetric for any value of $E_{r}$. When the trajectories (17) or (19) are used and $E_{r}=0, L_{j k}$ is symmetric as well. However, when the trajectories (17) or (19) are used and $E_{r} \neq 0$, the transport matrix defined in this manner is generally not symmetric.

Different definitions of the transport matrix have been given elsewhere in the literature, ${ }^{16}$ but the definition here has several nice properties. First, the matrix is dimensionless. Second, $L_{j k}$ is symmetric (in the cases described above). Third, $L_{j k}$ depends on the magnetic geometry and physical parameters only through $B / B_{0}, I / G, l$, a normalized collisionality

$$
\nu^{\prime}=\frac{(G+\imath I) \nu_{\mathrm{ii}}}{v_{\mathrm{i}} B_{0}},
$$

and a normalized electric field

$$
E_{*}=\frac{c G}{\imath v_{\mathrm{i}} B_{0}} \frac{d \Phi_{0}}{d \psi},
$$

and not on any other individual parameters such as density, temperature, $G$, etc. In (41), $\nu_{\mathrm{ii}}=4 \sqrt{2 \pi} n Z^{4} e^{4} \ln \Lambda /$ $\left(3 m^{1 / 2} T^{3 / 2}\right)$ is the ion-ion collision frequency. Typically, $I$ $\ll G$ and $G \approx B_{0} R$, where $R$ is the major radius of the device, so $\nu^{\prime} \approx \nu_{\mathrm{ii}} R / v_{\mathrm{i}}$. In axisymmetry, $E_{*}$ corresponds to the poloidal Mach number: $E_{*} \approx\left(B / B_{\mathrm{pol}}\right)\left|\boldsymbol{v}_{E 0}\right| / v_{\mathrm{i}}$, where $B_{\mathrm{pol}}$ is the poloidal magnetic field. Therefore, $E_{*}$ corresponds to the electric field normalized by the so-called resonant electric field ${ }^{16} E_{r}^{\text {res }}=r i v_{\mathrm{i}} B /(R c)$, with $r / R$ the inverse aspect ratio.

Several properties of the matrix $L_{j k}$ are noteworthy. Using the property $\int d^{3} v\left(g / F_{\mathrm{i}}\right) C_{\mathrm{ii}}\{g\} \leq 0$ for any $g$, which holds for all three ion-ion collision operators considered here, then $\operatorname{sgn}\left(L_{11}\right)=\operatorname{sgn}\left(L_{22}\right)=-\operatorname{sgn}\left(L_{33}\right)=-\operatorname{sgn}\left((G+l I) / B_{0}\right)$. This property holds when $E_{r}=0$, and it holds when $E_{r} \neq 0$ for the DKES trajectories, but it may not hold when $E_{r} \neq 0$ for the partial or full trajectories. Second, for all three trajectory models, the elements $L_{j k}$ are independent of the sign of the electric field: $L_{j k}\left(E_{*}\right)=L_{j k}\left(-E_{*}\right)$, assuming the stellarator symmetry property $B(\theta, \zeta)=B(-\theta,-\zeta)$ for some choice of the origin of $\theta$ and $\zeta$. This symmetry of $L_{j k}$ follows from a symmetry in the kinetic equation: if the signs of $\theta, \zeta, v_{\|}$, and $d \Phi_{0} / d \psi$ are all 
reversed in (16), the sign of $f_{i 1}$ will reverse, leaving the left-hand side of (40) unchanged.

\section{COMPARISON OF $E_{r}$ TERMS}

Figures 1 and 2 show a SFINCS computation of the ion transport matrix elements for the 3 trajectory models in two different stellarator geometries. The calculations in Figure 1 are performed for the $r / a=0.5$ surface of the LHD stellarator $^{41}$ in its standard configuration. (Here, the flux function $r$ is defined to be proportional to the square root of the toroidal flux enclosed by the flux surface in question.) The calculations in Figure 2 are performed for the $r / a=0.5$ surface of the W7-X stellarator ${ }^{42,43}$ in its standard configuration. In the LHD calculation, only the Boozer harmonics of $B(\theta, \zeta) / B_{0}$ with amplitude $>10^{-2}$ are retained, as listed in Table I of Ref. 4, whereas all harmonics with relative amplitude $>4 \times 10^{-5}$ are retained for the W7-X calculations. For both figures, the Fokker-Planck collision operator is used, and the collisionality is set to $\nu^{\prime}=0.01$. As both figures illustrate, the electric field has negligible effect on the transport matrix elements when $E_{*}<0.01$. For these small values of the electric field, the radial step size for diffusion is limited by collisions rather than by $\boldsymbol{E} \times \boldsymbol{B}$ precession. As $E_{r} \rightarrow 0$, all the matrix elements converge smoothly to their $E_{r}=0$ limits. For $E_{*}$ in the range [0.01, 0.3], the $\boldsymbol{E} \times \boldsymbol{B}$ precession suppresses radial transport, as can be seen by the reduction in $\left|L_{11}\right|$ and $\left|L_{22}\right|$. In this regime of $E_{*}$, the three trajectory models give nearly identical results for all the transport matrix elements. However, once $E_{*}$ exceeds about 0.3 , the results from the three trajectory models begin to separate.
In all probability, the reason why the three trajectory models agree so well with each other below the resonance is that they all capture the principal mechanism of transport in the $\sqrt{\nu}$-regime. The $\boldsymbol{E} \times \boldsymbol{B}$ drift convects most locally trapped particles poloidally around the torus, thus preventing them from drifting to the wall, and the transport is instead dominated by shallowly trapped and barely passing particles that are scattered back and forth across the trapped-passing boundary on a time scale equal to the poloidal convection time. ${ }^{3}$ This behavior is not likely to be affected by the approximations made in the DKES and partial trajectory models.

In the typical "ion root" scenario, $E_{*}$ can be estimated by noting that the ambipolar electric field arises to bring the ion particle transport down to the electron level, and is therefore approximately determined so as to reduce the magnitude of the thermodynamic force appearing as the first component of the vector on the right-hand side of (40). The electric field is thus of order $E_{r} \sim T /\left(e L_{\perp}\right)$, where $L_{\perp}$ denotes the length scale corresponding to the pressure gradient. It is thus expected that $E_{*}$ is of order $E_{*} \sim \rho_{\theta} / L_{\perp}$, where $\rho_{\theta}=\rho /(\imath \epsilon)$ and $\epsilon$ is the inverse aspect ratio, and the ratio $\rho_{\theta} / L_{\perp}$ is typically $\ll 1$. In W7-X, $E_{*}$ is predicted to be a few percent in normal plasma scenarios. ${ }^{44}$ The largest $E_{r}$ in normal W7-X scenarios is predicted to be a few tens of $\mathrm{kV} / \mathrm{m}$, in the edge where density gradients are steep, corresponding to $E_{*}$ up to a few tenths. ${ }^{44}$ However, in other previous experiments, scenarios with strong electron heating can cause $T_{e} \gg T_{i}$, giving rise to large positive "electron root" electric fields. ${ }^{45}$ In these scenarios, $E_{*}$ may be $\sim 1$.
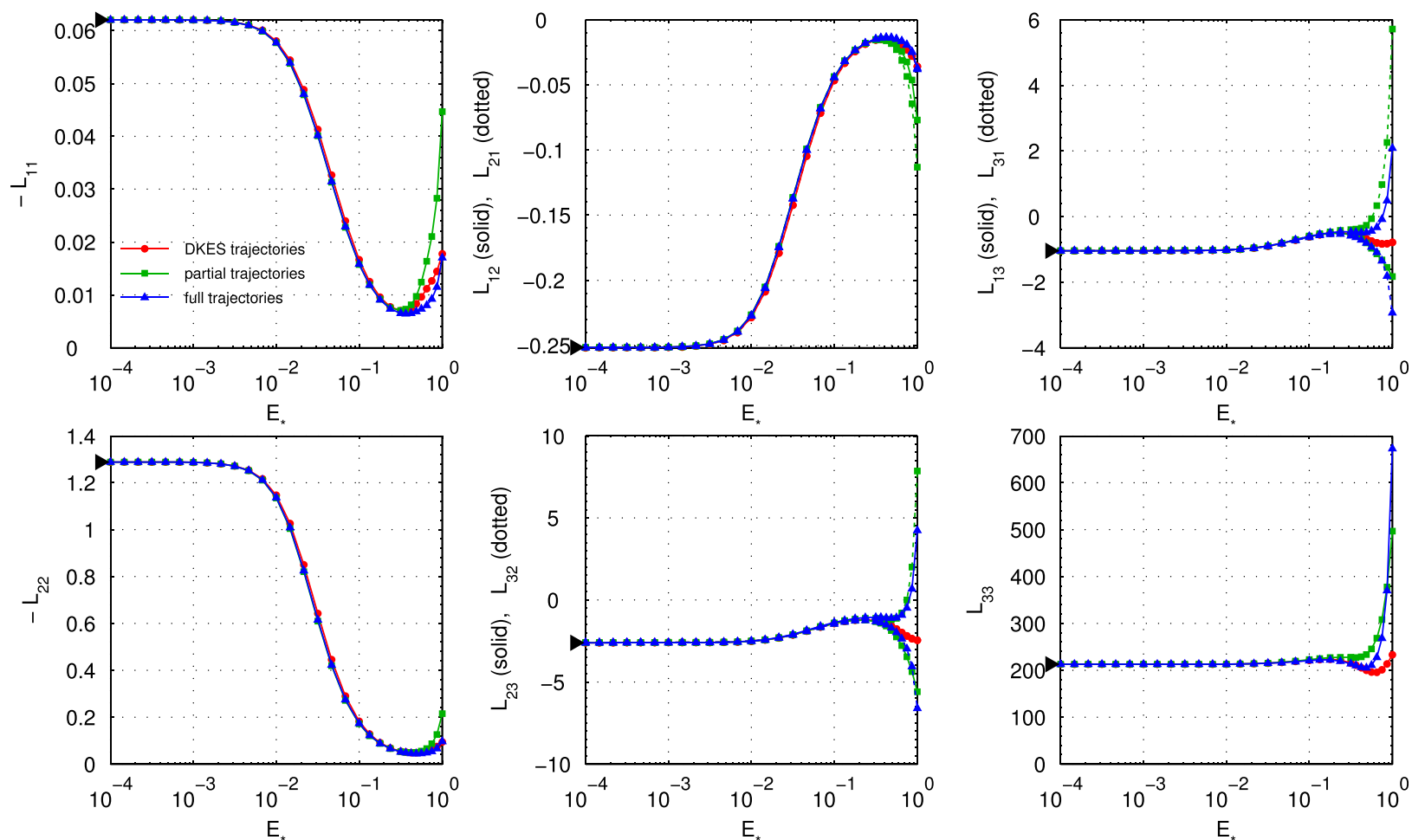

FIG. 1. Comparison of trajectory models for LHD standard geometry at $\nu^{\prime}=0.01$, using linearized Fokker-Planck collisions. The ion transport matrix elements (defined in (40)) are plotted as functions of the normalized radial electric field (42). Results for $E_{r}=0$ are indicated by the $>$ symbol to the left of each plot. 

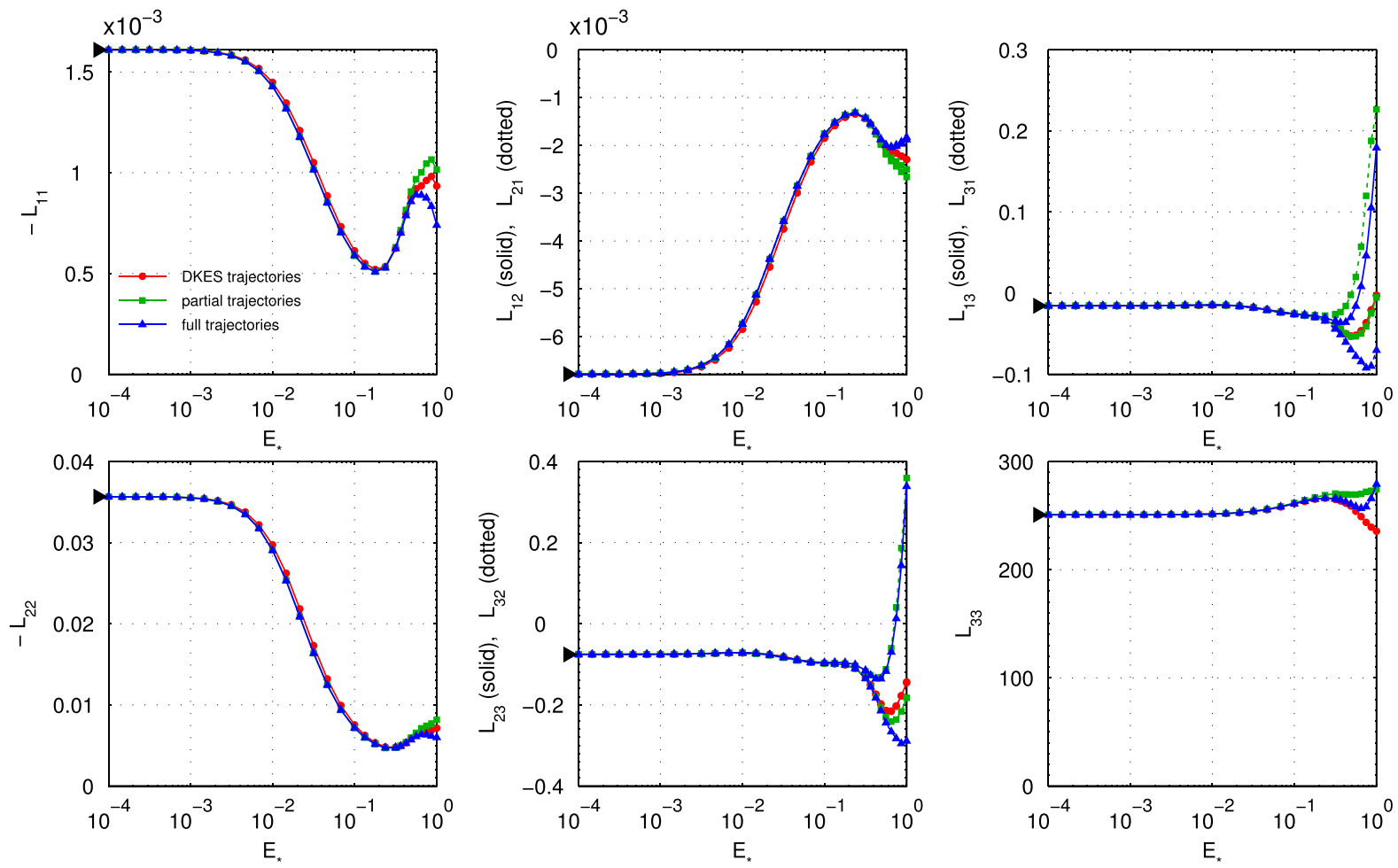

FIG. 2. Comparison of trajectory models for W7-X standard geometry at $\nu^{\prime}=0.01$, using linearized Fokker-Planck collisions. The ion transport matrix elements (defined in (40)) are plotted as functions of the normalized radial electric field (42). Results for $E_{r}=0$ are indicated by the symbol to the left of each plot.

Further analysis of whether the choice of trajectory model is significant in W7-X is shown in Figure 3. This calculation is based on the scenario considered in Figure 5 of Ref. 44. We focus on the radial location $r=0.45 \mathrm{~m}$ $(r / a=0.88)$ in which the pressure gradient is strong. This gradient should result in a large $E_{r}$, as predicted both by the argument in the preceding paragraph, and by the modeling in Ref. 44 based on incompressible- $\boldsymbol{E} \times \boldsymbol{B}$ computations. (Here, the flux label $r$ is defined by $\pi r^{2} B_{0}=2 \pi \psi$.) Matching the parameters in that work, we consider a pure hydrogen plasma with $n=6.6 \times 10^{19} \mathrm{~m}^{-3}, \quad T_{\mathrm{e}}=T_{\mathrm{i}}=1 \mathrm{keV}, \quad d n / d r=-1.2$ $\times 10^{21} \mathrm{~m}^{-4}$, and $d T_{\mathrm{e}} / d r=d T_{\mathrm{i}} / d r=-16 \mathrm{keV} / \mathrm{m}$. These values correspond to $\nu^{\prime}=0.03$ and $E_{r}^{\text {res }}=100 \mathrm{kV} / \mathrm{m}$. For this scenario, kinetic electrons are included in SFINCS along with the ions. Inter-species linearized Fokker-Planck collisions are included with no expansion in mass ratio.

The radial fluxes of ions and electrons as functions of $E_{r}$ are shown in Figure 3(a). The electron fluxes (dashed curves) are very small $\left(\sim \sqrt{m_{\mathrm{e}} / m_{\mathrm{i}}}\right)$ compared to the ion fluxes and are identical between the three trajectory models. No difference between the models is expected for the electrons, since $E_{*}$ defined using the electron rather than ion thermal speed is always $\ll 1$. The vertical magenta dotted line indicates the ambipolar value of $E_{r} \approx-33 \mathrm{kV} / \mathrm{m}$, which is effectively identical for the three trajectory models, and comparable to the value predicted in Ref. 44. This electric field is roughly one third of the resonant value, and therefore the ion transport coefficients are just beginning to separate for the three models. Heat fluxes are shown in Figure 3(b), showing similar behavior to the particle fluxes.

Figure 3(c) shows the surface-averaged ion parallel flow. At the ambipolar value of $E_{r}$, the three trajectory models yield similar values for the predicted flow. At lower magnitudes of $E_{r}$, the flows predicted by the three models are nearly indistinguishable. However, at larger electric fields, the three models begin to give quite different predictions. This change in behavior around $E_{*} \sim 0.3$ is consistent with the patterns in Figures 1 and 2. A similar pattern is visible in the bootstrap current density, shown in Figure 3(d). At the ambipolar value of $E_{r}$, the partial trajectory model predicts $27 \%$ more bootstrap current than the full trajectory model, and the DKES model predicts $8 \%$ more bootstrap current than the full trajectory model. Interestingly, if the electric field exceeds $60 \mathrm{kV} / \mathrm{m}$ in the inward (ion root) direction, the bootstrap current in the full trajectory model changes sign, whereas there is no sign change in the DKES model.

Figures 3(e) and 3(f) illustrate the ion particle and heat sources computed as part of the calculation. As expected, the particle and heat sources are zero for the DKES model; and for the full trajectory model, the particle source is always zero and the heat source vanishes at the ambipolar $E_{r}$. Electron sources are negligible. The plots show $S_{H \mathrm{p}}$ and $S_{H \mathrm{~h}}$ from (27) normalized to a gyro-Bohm transport time scale $t_{g B}=L^{2} / D_{g B}$ with $D_{g B}=\left(\rho_{i} / L\right) c T /\left(e B_{0}\right)$, thereby roughly normalizing the numerical sources to the scale of real physical sources arising from the divergence of the turbulent and neoclassical fluxes. For this comparison, we choose $L=-n /(d n / d r)$ to be the density scale length. For the range of electric fields considered, the numerical sources are small on this transport time scale, giving confidence in the model. For the parameters considered, $\nu_{\mathrm{ii}}$ $t_{g B}=0.4$, so dividing the values in Figures 3(e) and 3(f) by this factor, the source terms evidently remain much smaller 

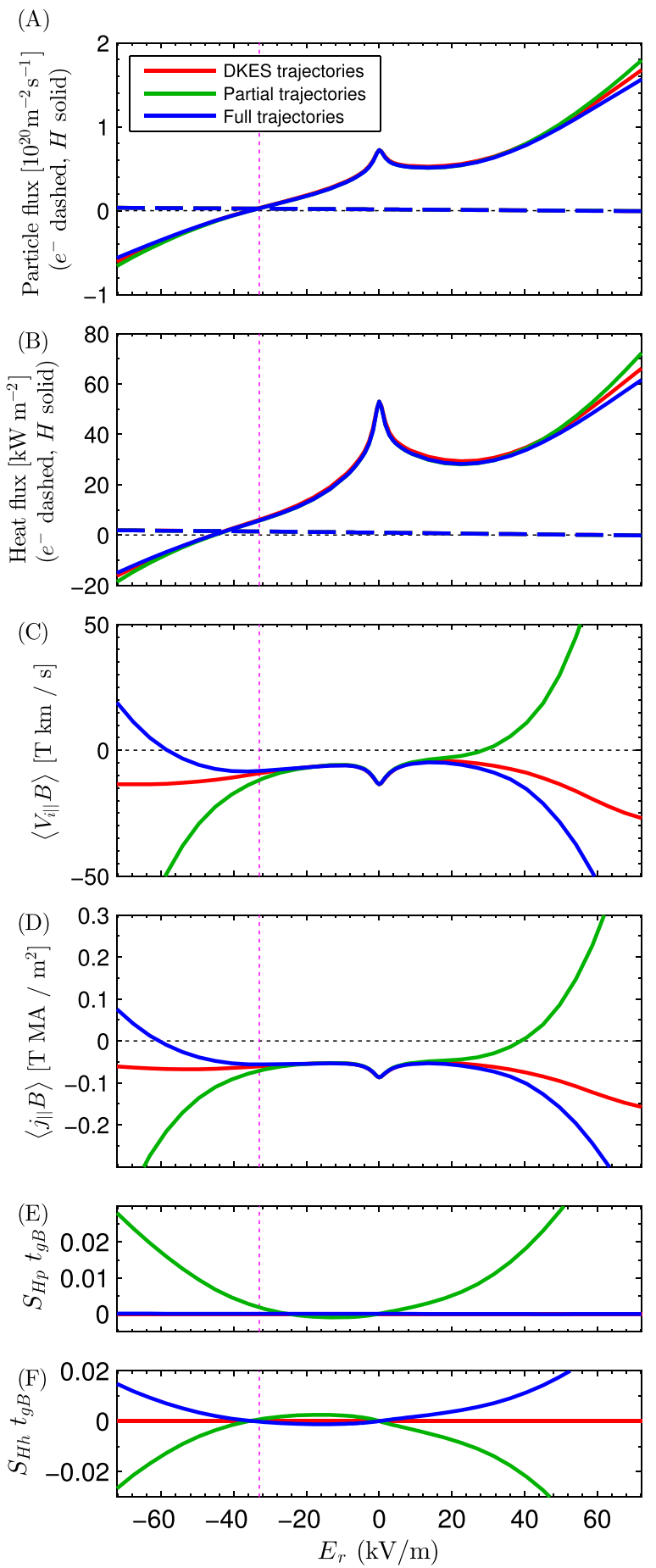

FIG. 3. Fluxes (A)-(B), flow (C), and bootstrap current (D) computed for a scenario of steep pressure gradient near the edge of W7-X. Magenta dotted line is the ambipolar $E_{r}$, effectively identical for the three trajectory models. The ion particle and heat sources in $(\mathrm{E})-(\mathrm{F})$ are normalized by a gyro-Bohm transport time $t_{g B}$.

than the collision term in the kinetic equation for this calculation.

\section{COMPARISON OF COLLISION OPERATORS}

Figures 4 and 5 show the transport matrix elements for the LHD and W7-X geometries described earlier, this time comparing the different collision operators as a function of collisionality. The comparison is done for $d \Phi_{0} / d \psi=0$, so the three trajectory models become identical, and the sources $S_{a}$ vanish. It can be seen in the figures that at high collisionality, momentum conservation is important for all the transport matrix elements (with the possible exception of $L_{22}$.) At low collisionality, momentum conservation is unimportant for $L_{11}, L_{12}, L_{21}$, and $L_{22}$. These matrix elements represent $1 / \nu$-regime radial transport (when $\nu^{\prime} \ll 1$ ), which is associated with pitch-angle scattering of helically trapped particles. Thus, the pitch-angle scattering approximation for collisions accurately captures the dominant physics in these cases. When $d \Phi_{0} / d \psi \neq 0$, the same is true for the $\sqrt{\nu}$-regime, where the main effect of the collisions is to scatter particles across a thin collisional boundary layer in velocity space around the trapped-passing boundary.

The other matrix elements $\left(L_{13}, L_{23}, L_{31}, L_{32}\right.$, and $\left.L_{33}\right)$ are more sensitive to momentum conservation at low collisionality. For all the matrix elements at all collisionalities, the momentum-conserving model operator reproduces all the trends of the more accurate linearized Fokker-Planck operator, though with some $O(1)$ differences.

Note in Figs. 4 and 5 that the scaling of the $L_{11}$ and $L_{12}$ coefficients at high collisionality depends crucially on whether momentum is conserved in the collision operator. In the momentum-conserving calculations, these transport coefficients are inversely proportional to $\nu$, whereas they are proportional to $\nu$ if the collisions are approximated by pure pitch-angle scattering. To understand why, it is useful to recall that the Pfirsch-Schlüter particle flux consists of two terms: one related to the parallel friction force and one related to parallel viscosity. ${ }^{46,47}$ This is most easily seen by taking the scalar product of the lowest-order plasma current, which satisfies $\boldsymbol{J} \times \boldsymbol{B}=c p^{\prime}(\psi) \nabla \psi$, with the momentum equation,

$$
\begin{aligned}
m_{a} n_{a} \boldsymbol{V}_{a} \cdot \nabla \boldsymbol{V}_{a}= & n_{a} e_{a}\left(-\nabla \Phi+c^{-1} \boldsymbol{V}_{a} \times \boldsymbol{B}\right) \\
& -\nabla p_{a}-\nabla \cdot \boldsymbol{\pi}_{a}+\boldsymbol{F}_{a},
\end{aligned}
$$

neglecting the left-hand side. Since $\nabla \cdot \boldsymbol{J}=0$ and $n_{a}$ is a flux function in lowest order, we obtain

$$
\left\langle n_{a} \boldsymbol{V}_{a} \cdot \nabla \psi\right\rangle=\frac{1}{e_{a} p^{\prime}(\psi)}\left\langle\boldsymbol{J} \cdot\left(\boldsymbol{F}_{a}-\nabla \cdot \boldsymbol{\pi}_{a}\right)\right\rangle,
$$

where the term corresponding to the perpendicular component of the friction force $\boldsymbol{F}_{a}$ represents the classical particle flux and the other terms the neoclassical flux,

$$
\left\langle n_{a} \boldsymbol{V}_{a} \cdot \nabla \psi\right\rangle_{n c}=\frac{1}{e_{a} p^{\prime}}\left\langle J_{\|} F_{a \|}+\pi_{a}: \nabla \boldsymbol{J}\right\rangle
$$

where the viscosity tensor is $\boldsymbol{\pi}_{a}=\left(p_{a \|}-p_{a \perp}\right)(\boldsymbol{b} \boldsymbol{b}-\boldsymbol{I} / 3)$. The first term in (45) is proportional to $\nu$ and therefore dominates at high collisionality, but vanishes when there is only a single ion species because of momentum conservation in like-particle collisions. All that remains is therefore the particle flux caused by parallel viscosity, which is inversely proportional to $\nu$ at high collisionality. ${ }^{31}$ In the pure pitchangle-scattering model, however, parallel momentum conservation is violated, leading to spurious friction-driven 

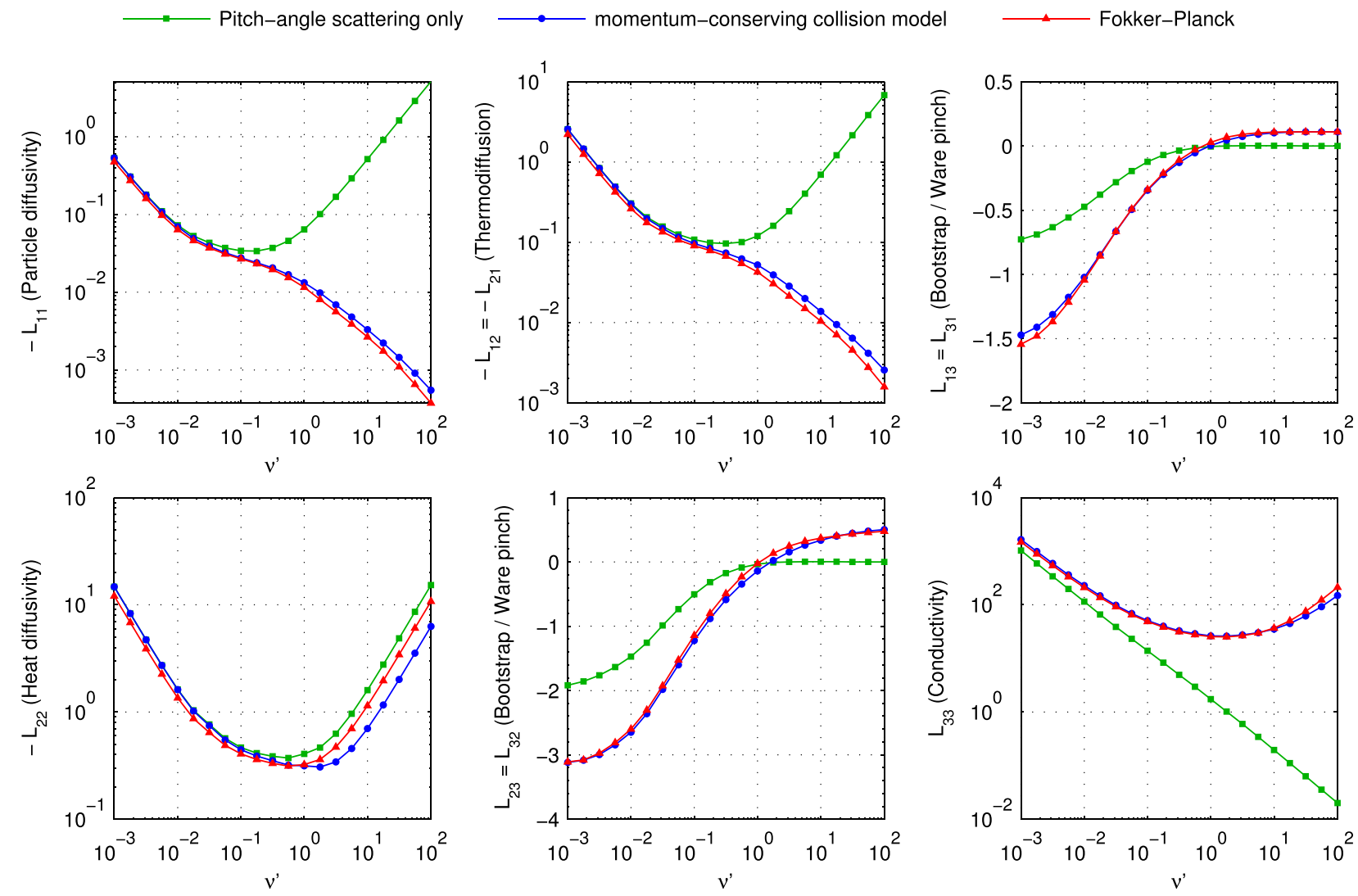

FIG. 4. The ion transport matrix elements (defined in (40)) are plotted as functions of the collisionality (41) for LHD geometry at $E_{r}=0$. SFINCS computations for three different collision operators are compared.

transport proportional to $\nu$. This is why the green curves have a slope of +1 for large $\nu$ in the logarithmic plots of $L_{11}$ and $L_{12}$ in Figures 4 and 5, while the blue and red curves have the slope -1 .

A similar difference between the momentum-conserving and pitch-angle-scattering operators is evident in the parallel conductivity coefficient $L_{33}$. The flow that arises in response to a parallel electric field is determined by the parallel momentum equation $\boldsymbol{b} \cdot(43)$, where the parallel friction force $F_{\| a}$ again vanishes when only a single ion species is considered. Hence

$$
n_{a} Z_{a} e\left\langle B E_{\|}\right\rangle=\left\langle\boldsymbol{B} \cdot\left(\nabla \cdot \pi_{a}\right)\right\rangle=\left\langle\left(p_{\perp}-p_{\|)} \nabla_{\|} B\right\rangle .\right.
$$

In the absence of radial gradients, the pressure anisotropy in the Pfirsch-Schlüter regime is proportional to the parallel flow velocity and inversely proportional to the collision frequency. ${ }^{46}$ The flow $\left\langle V_{\|} B\right\rangle$ is therefore proportional to $\nu$ in the Pfirsch-Schlüter regime unless momentum conservation is violated. In the latter case, the spurious friction force causes $\left\langle V_{\|} B\right\rangle$ to be inversely proportional to $\nu$, as can be seen in Figs. 4 and 5.

When $\nu^{\prime}<1$, the resolution required in the $\theta, \zeta$, and $\xi$ coordinates increases as $\nu^{\prime}$ decreases, due to the boundary layers that develop in phase space. The highest resolution used for results presented here, corresponding to the W7-X calculations at $\nu^{\prime}=10^{-3}$, was $N_{\theta}=29, N_{\zeta}=83, N_{\xi}=180$, and $N_{x}=5$, giving a $2166302 \times 2166302$ linear system. Here, $N_{j}$ is the number of grid points or modes in coordinate $j$.
Each calculation at this resolution with the Fokker-Planck collision operator required $\sim 30-50 \mathrm{~min}$ to run on 4 nodes of the Edison computer at NERSC. At higher collisionality, or if fewer harmonics are retained in $B(\theta, \zeta)$, lower resolution is sufficient, so memory and time requirements are reduced; for example, in the same W7-X geometry at $\nu^{\prime}=10^{-2}$, sufficient resolution parameters for convergence were $N_{\theta}=11, N_{\zeta}=64$, $N_{\xi}=100$, and $N_{x}=5$, and computations required 3 min on 1 node of Edison. Computations with $\nu^{\prime}>10^{-2}$ can typically be run on a laptop.

When the pure pitch-angle scattering collision operator and DKES trajectories are chosen, the kinetic equation solved in SFINCS becomes identical to the one solved in the DKES code. ${ }^{5,6}$ In this case, it was verified that the two codes agreed for all elements of the transport matrix, as demonstrated in Figure 5. For this figure, the monoenergetic transport coefficients computed by DKES have been integrated over velocity with the appropriate weights and normalized in the same way as (40).

In the short-mean-free-path limit $\nu^{\prime} \gg 1$, the ion transport and flow can be computed analytically in terms of the parallel current. ${ }^{46}$ The transport matrix elements associated with the Fokker-Planck collision operator may therefore be extracted from Ref. 46 and are summarized in Appendix B. Plotted in Figure 6 (dashed and dotted-dashed lines), these analytic high-collisionality limits agree quite well with the Fokker-Planck SFINCS computations in the appropriate $\nu^{\prime} \gg 1$ limit. 

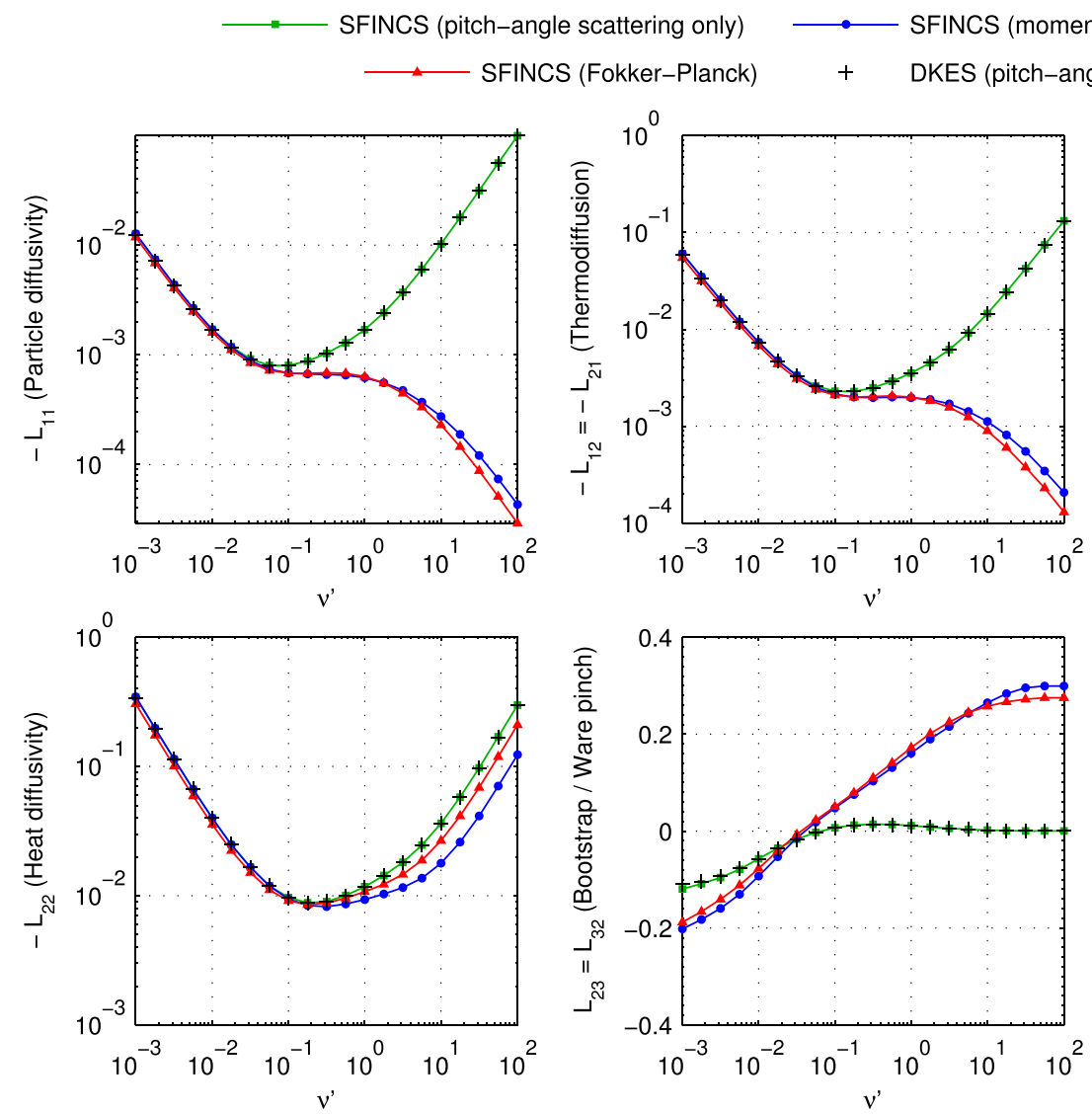
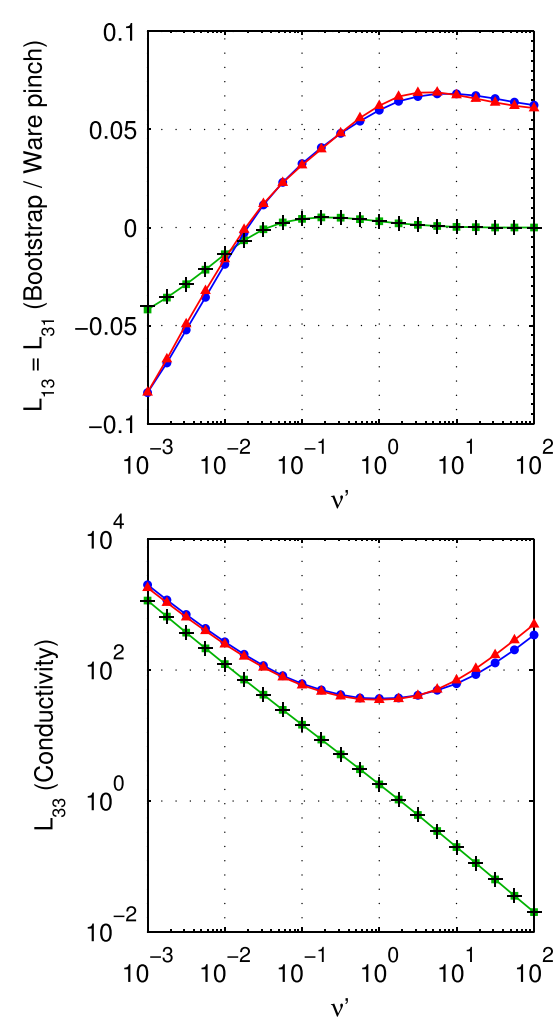

FIG. 5. The ion transport matrix elements (defined in (40)) are plotted as functions of the collisionality (41) for W7-X geometry at $E_{r}=0$. SFINCS computations for three different collision operators are compared. Also shown (black crosses) are the transport matrix elements computed using the DKES code ${ }^{5,6}$ which uses a pitch-angle scattering collision operator, demonstrating excellent agreement with SFINCS when the latter is run with the same collision model.

\section{DISCUSSION AND CONCLUSIONS}

In this work, we have examined the impact of several approximations made in stellarator kinetic codes, approximations related to the electric field and the collision operator. We have compared three versions of the drift-kinetic equation for a stellarator, consisting of (16) with the coefficients (17), (18), or (19). These three sets of expressions for $\dot{\boldsymbol{r}}_{a}, \dot{x}_{a}$, and $\dot{\xi}_{a}$ may be interpreted as effective particle trajectories (although we solve each form of the kinetic equation using continuum numerical methods). Equations (17) and (19) appear to be more accurate than (18); and as we have shown in Sec. IV, the full trajectory model (17) is the only one of the three models, which gives the correct parallel viscous force and which preserves intrinsic ambipolarity in quasisymmetry. However, as we have shown in Sec. III, the kinetic Eq. (16) with (17) or (19) is not well behaved when $E_{r} \neq 0$, with one or two unphysical constraints forced upon the distribution function. This analytic property of the kinetic equation must be dealt with before attempting to solve the equation numerically.

To eliminate this problem of unphysical constraints, we propose formulating the kinetic problem as in (36) with (27). A particle and heat source are introduced, along with the additional constraints that all the flux-surface- averaged density and pressure reside in the leading-order Maxwellian. For the model (18), the sources always vanish. For the model (17), the particle source vanishes for any $E_{r}$, and the energy source vanishes when $E_{r}$ takes on its ambipolar value. Equations (36) have been implemented in a new time-independent continuum code SFINCS, and the resulting ion transport matrices have been compared for the geometries of the LHD and W7-X stellarators. When $E_{r}$ is below roughly one-third of the resonant value, the three models give nearly indistinguishable results. This finding confirms that the incompressible- $\boldsymbol{E} \times \boldsymbol{B}$ trajectory model used in some $\operatorname{codes}^{5,6}$ is quite accurate in this small- $E_{r}$ regime, which is typically satisfied in experiments. Physically, the effect of $E_{r}$ in this regime is to generate a $\sqrt{\nu}$ regime of transport due to poloidal precession of helically trapped particles, and this process is retained (at least approximately) in all three trajectory models. Once $E_{r}$ approaches the resonance, however, the three trajectory models yield substantially different results. This $E_{r} \sim E_{r}^{\text {res }}$ regime can be relevant to experiments with high ratios $T_{\mathrm{e}} / T_{\mathrm{i}}$ (Refs. 23,45 , and 48) and strong gradients. ${ }^{49}$ In Figure 3, we find that in the large- $E_{r}$ region anticipated for the edge of $\mathrm{W} 7-\mathrm{X}$, the bootstrap current density in the full trajectory model is modestly reduced (by 8\%) compared to an incompressible- $\boldsymbol{E} \times \boldsymbol{B}$ calculation, but should larger values 

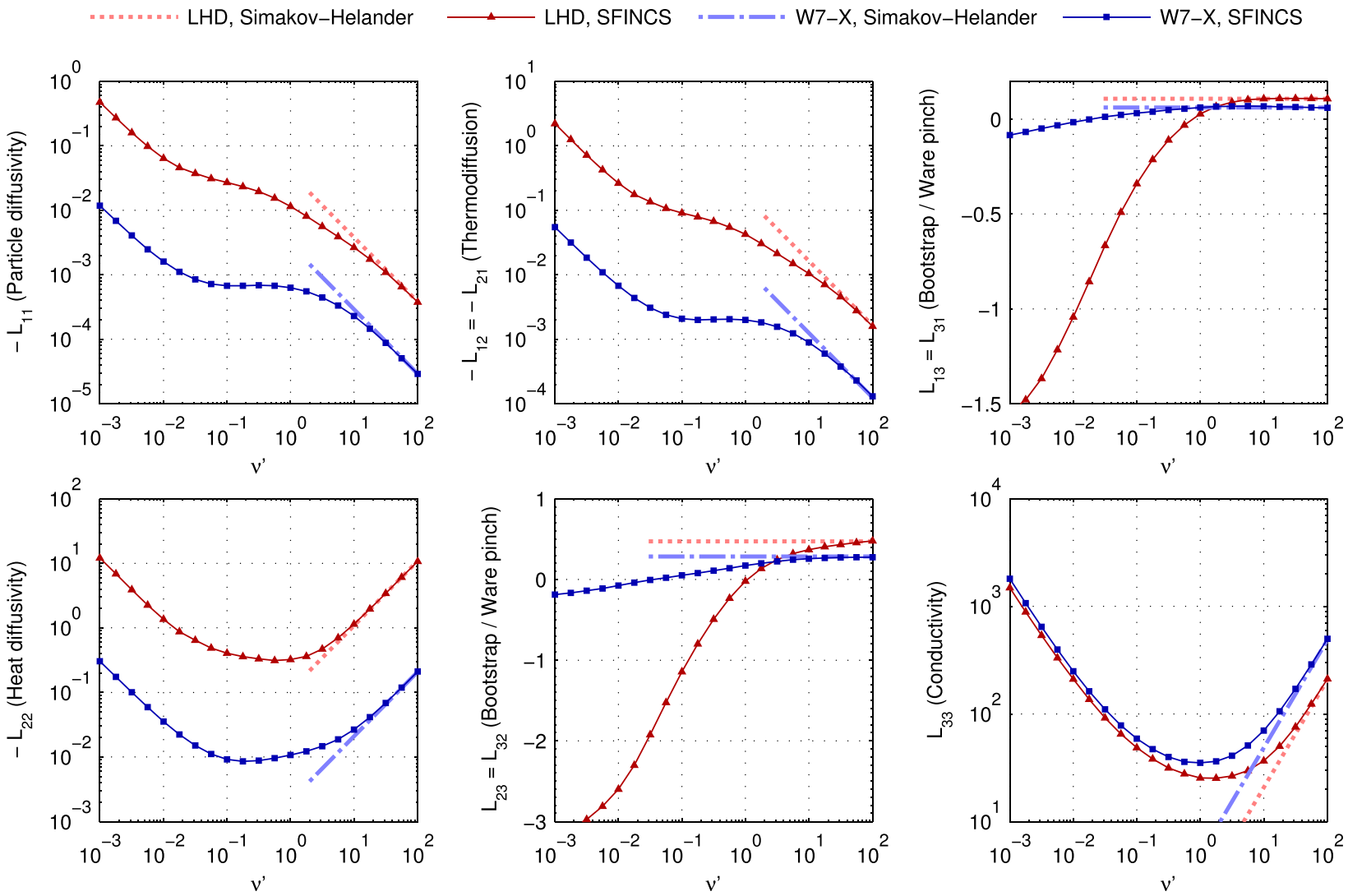

FIG. 6. The ion transport matrix elements (defined in (40)) are plotted as functions of the collisionality (41) for LHD and W7-X geometry at $E_{r}=0$. SFINCS results shown were computed using the linearized Fokker-Planck collision operator (so the solid curves here are identical to the red curves in Figures 4 and 5 .) Dashed and dotted-dashed lines indicate the analytic high-collisionality limits for Fokker-Planck collisions, discussed in Ref. 46 and in Appendix B, which agree quite well with the SFINCS computations at high collisionality.

of $E_{*}$ arise, we expect the deviation could grow more significant.

Since full coupling in the speed coordinate $x_{a}$ is retained in our numerical implementation, it is possible to directly compare results from the full linearized Fokker-Planck collision operator to results from simpler collision models. At low collisionality, the ion transport matrix elements $L_{11}, L_{12}$, $L_{21}$, and $L_{22}$ are nearly identical for the three collision models considered. This result makes sense physically since these matrix elements at low collisionality are associated with a piece of the distribution function that is localized to a narrow range of pitch angles, so pitch angle diffusion is the dominant collisional process. However, these same matrix elements at higher collisionality, or the other matrix elements at any collisionality, are sensitive to momentum conservation. The momentum-conserving model operator results in the correct scaling with collisionality when compared to the full linearized Fokker-Planck operator. However, there are still $O(1)$ differences in the transport coefficients computed with these two collision operators.

\section{ACKNOWLEDGMENTS}

This work was supported by the US Department of Energy through grants DE-FG02-91ER-54109 and DE-FG02-93ER-54197. M.L. is grateful to the Plasma Science and Fusion Center at the Massachusetts Institute of
Technology, where he carried out much of the code development, and for travel support from the Max-Planck-Institut für Plasmaphysik in Greifswald, Germany. Some of the computer simulations presented here used resources of the National Energy Research Scientific Computing Center (NERSC), which is supported by the Office of Science of the U.S. Department of Energy under Contract No. DE-AC0205CH11231. M.L. was supported by the Fusion Energy Postdoctoral Research Program administered by the Oak Ridge Institute for Science and Education. We are thankful to J. Geiger for providing the W7-X equilibrium data, and to Craig Beidler and Peter Catto for other input on this work. We also thank the anonymous referee for making several suggestions that substantially improved the paper.

\section{APPENDIX A: QUASISYMMETRY ISOMORPHISM}

A useful test of a stellarator neoclassical code such as the one described here is the quasisymmetry isomorphism, discussed analytically in Refs. 50-52. Equivalent to the definition at the end of Sec. IV, ${ }^{33}$ a quasisymmetric magnetic field is one satisfying $B(\theta, \zeta)=y(M \theta-N \zeta)$ for some periodic function $y$ and integers $M$ and $N$. Magnetic fields with the same $y$ but different $M$ and $N$ are said to be isomorphic in that the associated transport matrices must be related in the following manner. Suppose the transport matrices are computed for several quasisymmetric magnetic fields with different values of $M$ and $N$, varying the collision frequency in each calculation 
so $\nu_{\mathrm{ii}} /(I M-N)$ remains fixed, and varying the radial electric field so $\left(d \Phi_{0} / d \psi\right)(G M+I N) /(\imath M-N)$ remains fixed. In such a scan of $M$ and $N$, it can be shown analytically ${ }^{50-52}$ that the transport matrix elements should vary as follows: $L_{11}, L_{12}$, $L_{21}$, and $L_{22}$ vary $\propto(N I+M G)^{2} /(\imath M-N) ; L_{13}, L_{23}, L_{31}$, and $L_{32}$ vary $\propto(N I+M G) /(l M-N)$; and $L_{33}$ varies $\propto 1 /(\imath M-N)$. This isomorphism holds for all the trajectory models considered in this paper.

As $\nu_{\mathrm{ii}} /(\imath M-N)$ is to be held fixed in this test, while $\imath M-N$ can change sign as $M$ and $N$ are varied, the collision frequency to use can be negative. While $\nu_{\mathrm{ii}}<0$ does not make sense physically, it poses no mathematical or numerical problem. In any stellarator (even a non-quasisymmetric and/or non-stellarator-symmetric one), if the signs of the collision frequency, $\xi$, and $d \Phi_{0} / d \psi$ are simultaneously reversed in the kinetic equation, the part of $f_{i 1}$ driven by $\left\langle E_{\|} B\right\rangle$ remains unchanged, while the part driven by radial gradients changes sign. Thus, $L_{11}, L_{12}, L_{21}, L_{22}$, and $L_{33}$ change sign, while $L_{13}, L_{23}, L_{31}$, and $L_{32}$ remain unchanged. Therefore, another way to express the quasisymmetry isomorphism (even for non-stellarator-symmetric $y$ ) that preserves $\nu_{\mathrm{ii}}>0$ is the following: if $M$ and $N$ are varied holding $\nu_{\mathrm{ii}} /|l M-N|$ and $\left(d \Phi_{0} / d \psi\right)(G M+I N) /|\imath M-N|$ fixed, $L_{11}, L_{12}, L_{21}$, and $L_{22}$ should vary $\propto(N I+M G)^{2} /|\imath M-N| ; L_{13}, L_{23}, L_{31}$, and $L_{32}$ should vary $\propto(N I+M G) /(\imath M-N)$; and $L_{33}$ should vary $\propto 1 /|\imath M-N|$.

It was verified that the SFINCS code obeyed both versions of these isomorphism transformations for various $y$, collisionality regimes, radial electric fields, trajectory models, and collision operators.

\section{APPENDIX B: ION TRANSPORT MATRIX AT HIGH COLLISIONALITY}

From the analytic calculations presented in Ref. 46, we can derive expressions for $L_{j k}$ of Eq. (40) in the PfirschSchlüter regime. Note that a pure plasma with singly charged ions is assumed in Ref. 46, so we specialize to this case of $Z=1$ in this appendix. The transport matrix elements depend on the function $u$ given by the solution to Eq. (8) in Ref. 46; $u$ is proportional to the parallel current divided by $B$. All coefficients but $L_{33}$ are straightforwardly obtained from Eqs. (14), (18), and (26) in Ref. 46 for the radial ion heat flux, the parallel ion flow, and the radial current, respectively, by suitable choices of the thermodynamic forces in the right-handside of Eq. (40) and using the symmetry of the transport matrix. To find the parallel conductivity coefficient $L_{33}$, we substitute the pressure anisotropy, given by Eq. (20) in Ref. 46, into Eq. (46) (of this paper) in the absence of radial gradients (i.e., when $E_{\|}$is the only thermodynamic force present). Then, $L_{33}$ can be found from the flow $\left\langle V_{\|} B\right\rangle$.

Expressions for the matrix coefficients in the PfirschSchlüter regime are summarized in Eqs. (B1) and (B2). Three numerical coefficients in the function $K_{2}^{\text {Simakov }}(\psi)$ arise from generalized Spitzer problems, which were solved in Ref. 46 by keeping a small number of Laguerre polynomials in kinetic energy. When these generalized Spitzer problems are solved keeping more energy polynomials, we obtain the more accurate coefficients given in $K_{2}$ below.

$$
\begin{aligned}
& L_{11}=0.96 \cdot 2^{1 / 2} \cdot \frac{3}{4} \frac{(G+l I)^{2}}{l^{2} G^{2}} G_{1}(\psi) \frac{1}{\nu^{\prime}}, \\
& L_{12}=L_{21}=0.96 \cdot 2^{1 / 2} \frac{(G+l I)^{2}}{l^{2} G^{2}} \\
& {\left[3.245 G_{1}(\psi)+0.085 G_{2}(\psi)\right] \frac{1}{\nu^{\prime}},} \\
& L_{13}=L_{31}=\frac{\left\langle u B^{2}\right\rangle}{G l}-\frac{\left\langle B^{2}\right\rangle}{G l} K_{1}(\psi), \\
& L_{22}=2^{1 / 2} \cdot \frac{8}{5} \frac{1}{l^{2} G^{2}} B_{0}^{2} H(\psi) \nu^{\prime}, \\
& L_{23}=L_{32}=\frac{1}{l G}\left[\frac{5}{2}\left\langle u B^{2}\right\rangle-\frac{5}{2} K_{1}(\psi)\left\langle B^{2}\right\rangle+K_{2}(\psi)\left\langle B^{2}\right\rangle\right] \text {, } \\
& L_{33}=\frac{1}{3 \cdot 0.96 \cdot 2^{1 / 2}} \frac{1}{(G+l I)^{2}} \frac{\left\langle B^{2}\right\rangle^{2}}{\left\langle\left(\nabla_{\|} B\right)^{2}\right\rangle} \nu^{\prime}, \\
& G_{1}(\psi)=\frac{\left\langle\left(\nabla_{\|} \ln B\right) \nabla_{\|}\left(u B^{2}\right)\right\rangle^{2}}{\left\langle\left(\nabla_{\|} B\right)^{2}\right\rangle}-\left\langle\left[\frac{\nabla_{\|}\left(u B^{2}\right)}{B}\right]^{2}\right\rangle, \\
& G_{2}(\psi)=\left\langle u\left(\nabla_{\|} \ln B\right) \nabla_{\|}\left(u B^{2}\right)\right\rangle \\
& -\frac{\left\langle\left(\nabla_{\|} \ln B\right) \nabla_{\|}\left(u B^{2}\right)\right\rangle\left\langle u\left(\nabla_{\|} B\right)^{2}\right\rangle}{\left\langle\left(\nabla_{\|} B\right)^{2}\right\rangle}, \\
& K_{1}(\psi)=\frac{\left\langle\left(\nabla_{\|} \ln B\right) \nabla_{\|}\left(u B^{2}\right)\right\rangle}{2\left\langle\left(\nabla_{\|} B\right)^{2}\right\rangle}, \\
& K_{2}(\psi)=1.97213 \frac{\left\langle u B^{2}\right\rangle}{\left\langle B^{2}\right\rangle}-1.03287 \cdot 2 K_{1}(\psi) \\
& +0.09361 \frac{\left\langle u\left(\nabla_{\|} B\right)^{2}\right\rangle}{\left\langle\left(\nabla_{\|} B\right)^{2}\right\rangle}, \\
& K_{2}^{\text {Simakov }}(\psi)=1.77 \frac{\left\langle u B^{2}\right\rangle}{\left\langle B^{2}\right\rangle}-0.91 \cdot 2 K_{1}(\psi) \\
& +0.05 \frac{\left\langle u\left(\nabla_{\|} B\right)^{2}\right\rangle}{\left\langle\left(\nabla_{\|} B\right)^{2}\right\rangle}, \\
& H(\psi)=\frac{\left\langle u B^{2}\right\rangle^{2}}{\left\langle B^{2}\right\rangle}-\left\langle u^{2} B^{2}\right\rangle .
\end{aligned}
$$

${ }^{1}$ G. Kagan and P. J. Catto, Plasma Phys. Controlled Fusion 52, 055004 (2010).

${ }^{2}$ A. A. Galeev, R. Z. Sagdeev, H. P. Furth, and M. N. Rosenbluth, Phys, Rev. Lett. 22, 511 (1969).

${ }^{3}$ D. D.-M. Ho and R. M. Kulsrud, Phys. Fluids 30, 442 (1987).

${ }^{4}$ C. D. Beidler, K. Allmaier, M. Y. Isaev, S. V. Kasilov, W. Kernbichler, G. O. Leitold, H. Maassberg, D. R. Mikkelsen, S. Murakami, M. Schmidt, D. A. Spong, V. Tribaldos, and A. Wakasa, Nucl. Fusion 51, 076001 (2011).

${ }^{5}$ S. P. Hirshman, K. C. Shaing, W. I. van Rij, C. O. Beasley, Jr., and E. C. Crume, Jr., Phys. Fluids 29, 2951 (1986).

${ }^{6}$ W. I. van Rij and S. P. Hirshman, Phys. Fluids B 1, 563 (1989).

${ }^{7}$ C. D. Beidler and W. D. D'haeseleer, Plasma Phys. Controlled Fusion 37, 463 (1995).

${ }^{8}$ V. Tribaldos, Phys. Plasmas 8, 1229 (2001).

${ }^{9}$ D. A. Spong, Phys. Plasmas 12, 056114 (2005).

${ }^{10}$ W. Kernbichler, S. V. Kasilov, G. O. Leitold, V. V. Nemov, and K. Allmaier, in 33rd EPS Conference on Plasma Phys., Rome (2006), p. 30I.

${ }^{11}$ S. Satake, R. Kanno, and H. Sugama, Plasma Fusion Res. 3, S1062 (2008).

${ }^{12}$ S. Satake, Y. Idomura, H. Sugama, and T.-H. Watanabe, Comput. Phys. Commun. 181, 1069 (2010).

${ }^{13}$ J. M. García-Regaña, R. Kleiber, C. D. Beidler, Y. Turkin, H. Maassberg, and P. Helander, Plasma Phys. Controlled Fusion 55, 074008 (2013). 
${ }^{14}$ P. Helander, Phys. Plasmas 14, 104501 (2007).

${ }^{15}$ H. Sugama, T. H. Watanabe, M. Nunami, and S. Nishimura, Plasma Phys. Controlled Fusion 53, 024004 (2011).

${ }^{16}$ C. D. Beidler, M. Y. Isaev, S. V. Kasilov, W. Kernbichler, H. Maassberg, S. Murakami, V. V. Nemov, and D. A. S. V. Tribaldos, in Proceedings of the 17th International Toki Conference and 16th International Stellarator/Heliotron Workshop, Toki (2007).

${ }^{17}$ M. Landreman, Plasma Phys. Controlled Fusion 53, 082003 (2011).

${ }^{18}$ O. Sauter, R. W. Harvey, and F. L. Hinton, Contrib. Plasma Phys. 34, 169 (1994).

${ }^{19}$ O. Sauter, C. Angioni, and Y. R. Lin-Liu, Phys. Plasmas 6, 2834 (1999).

${ }^{20}$ S. K. Wong and V. S. Chan, Plasma Phys. Controlled Fusion 53, 095005 (2011).

${ }^{21}$ E. A. Belli and J. Candy, Plasma Phys. Controlled Fusion 54, 015015 (2012).

${ }^{22}$ M. Landreman and D. R. Ernst, J. Comput. Phys. 243, 130 (2013).

${ }^{23}$ A. Briesemeister, K. Zhai, D. T. Anderson, F. S. B. Anderson, and J. N. Talmadge, Plasma Phys. Controlled Fusion 55, 014002 (2013).

${ }^{24}$ M. Taguchi, Phys. Fluids B 4, 3638 (1992).

${ }^{25}$ H. Sugama and S. Nishimura, Phys. Plasmas 9, 4637 (2002).

${ }^{26}$ H. Maassberg, C. D. Beidler, and Y. Turkin, Phys. Plasmas 16, 072504 (2009).

${ }^{27}$ R. D. Hazeltine, Plasma Phys. 15, 77 (1973).

${ }^{28}$ M. Landreman and D. R. Ernst, Plasma Phys. Controlled Fusion 54, 115006 (2012)

${ }^{29}$ M. Landreman and P. J. Catto, Plasma Phys. Controlled Fusion 55, 095017 (2013).

${ }^{30}$ M. N. Rosenbluth, W. M. MacDonald, and D. L. Judd, Phys. Rev. 107, 1 (1957).

${ }^{31}$ P. Helander and D. J. Sigmar, Collisional Transport in Magnetized Plasmas (Cambridge University Press, Cambridge, 2002).

${ }^{32}$ A. N. Simakov and P. J. Catto, Phys. Plasmas 12, 012105 (2005).

${ }^{33}$ P. Helander and A. N. Simakov, Phys. Rev. Lett. 101, 145003 (2008).

${ }^{34}$ M. Landreman, F. I. Parra, P. J. Catto, D. R. Ernst, and I. Pusztai, Plasma Phys. Controlled Fusion 56, 045005 (2014).

${ }^{35}$ S. Balay, J. Brown, K. Buschelman, W. D. Gropp, D. Kaushik, M. G. Knepley, L. C. McInnes, B. F. Smith, and H. Zhang, "PETSc Web Page," 2012, http://www.mcs.anl.gov/petsc.
${ }^{36}$ S. Balay, J. Brown, K. Buschelman, V. Eijkhout, W. D. Gropp, D. Kaushik, M. G. Knepley, L. C. McInnes, B. F. Smith, and H. Zhang, "PETSc users manual," Tech. Rep. ANL-95/11-Revision 3.3 (Argonne National Laboratory, 2012).

${ }^{37}$ Y. Saad and M. H. Schultz, SIAM J. Sci. Stat. Comput. 7, 856 (1986).

${ }^{38}$ G. L. G. Sleijpen and D. R. Fokkema, Electr. Trans. Numer. Anal. 1, 11 (1993).

${ }^{39}$ X. Li, J. Demmel, J. Gilbert, L. Grigori, M. Shao, and I. Yamazaki, "SuperLU Users' Guide," Tech. Rep. LBNL-44289 (Lawrence Berkeley National Laboratory, 1999), http://crd.lbl.gov/ xiaoye/SuperLU/.

${ }^{40}$ X. S. Li and J. W. Demmel, ACM Trans. Math. Software 29, 110 (2003)

${ }^{41}$ A. Iiyoshi, M. Fujiwara, O. Motojima, N. Ohyabu, and K. Yamazaki, Fusion Tech. 17, 169 (1990).

${ }^{42}$ C. D. Beidler, G. Grieger, F. Herrnegger, W. Harmeyer, J. Kisslinger, W. Lotz, H. Maassberg, P. Merkel, J. Nühenberg, F. Rau, J. Sapper, F. Sardei, R. Scardovelli, A. Schlüter, and H. Wobig, Fusion Tech. 17, 148 (1990).

${ }^{43}$ G. Grieger, C. D. Beidler, E. Harmeyer, W. Lotz, J. Kisslinger, P. Merkel, J. Nührenberg, F. Rau, E. Strumberger, and H. Wobig, Fusion Tech. 21, 1767 (1992).

${ }^{44}$ Y. Turkin, C. D. Beidler, H. Maassberg, S. Murakami, V. Tribalos, and A. Wakasa, Phys. Plasmas 18, 022505 (2011).

${ }^{45}$ M. Yokoyama, H. Maassberg, C. D. Beidler, V. Tribaldos, K. Ida, T. Estrada, F. Castejon, A. Fujisawa, T. Minami, T. Shimozuma, Y. Takeiri, A. Dinklage, S. Murakami, and H. Yamada, Nucl. Fusion 47, 1213 (2007).

${ }^{46}$ A. N. Simakov and P. Helander, Phys. Plasmas 16, 042503 (2009).

${ }^{47}$ S. Braun and P. Helander, Phys. Plasmas 17, 072514 (2010).

${ }^{48}$ J. Lore, W. Guttenfelder, A. Briesemeister, D. T. Anderson, F. S. B. Anderson, C. B. Deng, K. M. Likin, D. A. Spong, J. N. Talmadge, and K. Zhai, Phys. Plasmas 17, 056101 (2010).

${ }^{49}$ J. Baldzuhn, M. Kick, H. Maassberg, and W7-AS Team, Plasma Phys. Controlled Fusion 40, 967 (1998).

${ }^{50}$ A. Pytte and A. H. Boozer, Phys. Fluids 24, 88 (1981).

${ }^{51}$ A. H. Boozer, Phys. Fluids 26, 496 (1983).

${ }^{52}$ M. Landreman and P. J. Catto, Plasma Phys. Controlled Fusion 53, 015004 (2011) 